\title{
Exponential Stability of Periodic Solutions for Inertial Type BAM Cohen-Grossberg Neural Networks
}

\author{
Chunfang Miao and Yunquan Ke \\ Department of Mathematics, Shaoxing University, Shaoxing, Zhejiang 312000, China \\ Correspondence should be addressed to Yunquan Ke; keyunquan@usx.edu.cn
}

Received 6 February 2014; Revised 22 March 2014; Accepted 3 April 2014; Published 19 May 2014

Academic Editor: Zidong Wang

Copyright (C) 2014 C. Miao and Y. Ke. This is an open access article distributed under the Creative Commons Attribution License, which permits unrestricted use, distribution, and reproduction in any medium, provided the original work is properly cited.

\begin{abstract}
The existence and exponential stability of periodic solutions for inertial type BAM Cohen-Grossberg neural networks are investigated. First, by properly choosing variable substitution, the system is transformed to first order differential equation. Second, some sufficient conditions that ensure the existence and exponential stability of periodic solutions for the system are obtained by constructing suitable Lyapunov functional and using differential mean value theorem and inequality technique. Finally, two examples are given to illustrate the effectiveness of the results.
\end{abstract}

\section{Introduction}

The Cohen-Grossberg-type BAM neural networks model is initially proposed by Cohen and Grossberg [1], has their promising potential for the tasks of parallel computation, associative memory, and has great ability to solve difficult optimization problems. Thus, the analysis of the dynamical behaviors of bidirectional associative memory neural networks and Cohen-Grossberg neural networks is important and necessary. In recent years, many researchers have studied the stability and other dynamical behaviors of the CohenGrossberg-type BAM neural networks; see [2-10].

On the other hand, some authors studied neural networks, added the inertia, and obtained some results. For example, Li et al. [11] added the inertia to a delay differential equation which can be described by

$$
\ddot{x}=a \dot{x}-b x+c f(x-h x(t-\tau))
$$

and obtained obvious chaotic behavior. Liu et al. [12, 13] found chaotic behavior of the inertial two-neuron system with time through numerical simulation and gave that the system will lose its stability when the time delay is increased and will rise a quasiperiodic motion and chaos under the interaction of the periodic excitation. Wheeler and Schieve [14] added the inertia to a continuous-time Hopfield effective-neuron system which is shown to exhibit chaos. They explain that the chaos is confirmed by Lyapunov exponents, power spectra, and phase space plotsthis system is described by

$$
\begin{aligned}
& \ddot{x}_{1}=-a_{11} \dot{x}_{1}-a_{12} x_{1}+a_{13} \tanh \left(x_{1}\right)+a_{14} \tanh \left(x_{2}\right), \\
& \ddot{x}_{2}=-b_{11} \dot{x}_{2}-b_{12} x_{2}+b_{13} \tanh \left(x_{1}\right)+b_{14} \tanh \left(x_{2}\right) .
\end{aligned}
$$

Babcock and Westervelt [15] studied the electronic neural networks with added inertia and found that when the neuron couplings are of an inertial nature, the dynamics can be complex, in contrast to the simpler behavior displayed when they of the standard resistor-capacitor variety. For various values of the neuron gain and the quality factor of the couplings, they find ringing about the stationary points, instability and spontaneous oscillation, intertwined basins of attraction, and chaotic response to a harmonic drive. Ge and $\mathrm{Xu}[16]$ considered an inertial four-neuron delayed bidirectional associative memory model. Weak resonant double Hopf bifurcations are completely analyzed in the parameter space of the coupling weight and the coupling delay by the perturbation-incremental scheme. Others, Liu et al. [17, 18], investigated the Hopf bifurcation and dynamics of an inertial two-neuron system or in a single inertial neuron mode. Zhao et al. [19] investigated the stability and the bifurcation of a class of inertial neural networks. The authors Ke and Miao $[20,21]$ investigated stability of equilibrium point and periodic solutions in inertial BAM neural networks with 
time delays, respectively. From the above, the inertia can be considered a useful tool that is added to help in the generation of chaos in neural systems. Horikawa and Kitajima [22] investigated a kinematical description of traveling waves of the oscillations in neural networks with inertia. When the inertia is below a critical value and the state of each neuron is overdamped, properties of the networks are the same as those without inertia. The duration of the transient oscillations increases with inertia, and the increasing rate of the logarithm of the duration becomes more than double. When the inertia exceeds a critical value and the state of each neuron becomes underdamped, properties of the networks qualitatively change. The periodic solution is stabilized through the pitchfork bifurcation as inertia increases. More bifurcations occur so that various periodic solutions are generated, and the stability of the periodic solutions changes alternately. Ke and Miao [23] investigated the stability of inertial Cohen-Grossberg-type neural networks with time delays. To the best of our knowledge, the question on the periodic solutions of inertial type BAM Cohen-Grossberg neural networks with time delays is still open. To provide the theoretical basis of practical application, this paper is devoted to present a sufficient criterion to ensure the existence and exponential stability of periodic solutions for inertial type BAM Cohen-Grossberg neural networks with time delays.

We consider the following inertial type BAM CohenGrossberg neural networks with time delays:

$$
\begin{aligned}
\frac{d^{2} u_{i}(t)}{d t^{2}}= & -\alpha_{i} \frac{d u_{i}(t)}{d t}-a_{i}\left(u_{i}(t)\right) \\
\times & {\left[b_{i}\left(u_{i}(t)\right)-\sum_{j=1}^{m} c_{i j} f_{j}\left(v_{j}\left(t-\tau_{i j}(t)\right)\right)\right.} \\
& \left.-I_{i}(t)\right], \\
\frac{d^{2} v_{j}(t)}{d t^{2}=}- & \beta_{j} \frac{d v_{j}(t)}{d t}-d_{j}\left(v_{j}(t)\right) \\
\times & {\left[e_{j}\left(v_{j}(t)\right)-\sum_{i=1}^{n} h_{j i} g_{i}\left(u_{i}\left(t-\sigma_{j i}(t)\right)\right)\right.} \\
& \left.\quad-J_{j}(t)\right],
\end{aligned}
$$

for $i=1,2, \ldots, n, j=1,2, \ldots, m$, where the second derivative is called an inertial term of system (3); $\alpha_{i}, \beta_{j}>0$ are constants; $u_{i}(t)$ and $v_{j}(t)$ are the states of the $i$ th neuron from the neural field $F_{U}$ and the $j$ th neuron from the neural field $F_{V}$ at the time $t$, respectively; $f_{j}, g_{i}$ denote the activation functions of $j$ th neuron from $F_{V}$ and the $i$ th neuron from $F_{U}$, respectively; $c_{i j}$ weights the strength of the $i$ th neuron on the $j$ th neuron at the time $t-\tau_{i j}(t) ; h_{j i}$ weights the strength of the $j$ th neuron on the $i$ th neuron at the time $t-\sigma_{j i}(t)$; $0 \leq \tau_{i j}(t) \leq \tau$ and $0 \leq \sigma_{j i}(t) \leq \sigma ; I_{i}(t), J_{j}(t)$ denote the external inputs on the $i$ th neuron from $F_{U}$ and the $j$ th neuron from $F_{V}$ at the time $t$, respectively; $a_{i}\left(u_{i}(t)\right)$ and $d_{j}\left(v_{j}(t)\right)$ represent amplification functions; $b_{i}\left(u_{i}(t)\right)$ and $e_{j}\left(v_{j}(t)\right)$ are appropriately behaved functions such that the solutions of model (3) remain bounded.

The initial conditions of system (3) are given by

$$
\begin{aligned}
& u_{i}(s)=\varphi_{u i}(s), \quad \frac{d u_{i}(s)}{d t}=\psi_{u i}(s), \quad-\tau \leq s \leq 0, \\
& v_{j}(s)=\varphi_{v j}(s), \quad \frac{d v_{j}(s)}{d t}=\psi_{v j}(s), \quad-\sigma \leq s \leq 0,
\end{aligned}
$$

where $\varphi_{u i}(s), \psi_{u i}(s), \varphi_{v j}(s)$, and $\psi_{v j}(s)$ are bounded and continuous functions.

This paper is organized as follows. Some preliminaries are given in Section 2. In Section 3, the sufficient conditions are derived which ensure the existence and exponential stability of periodic solutions for inertial Cohen-Grossberg-type BAM neural networks. In Section 4, two illustrative examples are given to show the effectiveness of the proposed theory.

\section{Preliminaries}

Throughout this paper, we make the following assumptions.

$\left(H_{1}\right)$ For each $i=1,2, \ldots, n, j=1,2, \ldots, m$, the functions $a_{i}(u), d_{j}(v), b_{i}(u)$, and $e_{j}(v)$ are differentiable and satisfy

$$
\begin{gathered}
0<\underline{a}_{i} \leq a_{i}(u) \leq \bar{a}_{i}, \quad\left|a_{i}^{\prime}(u)\right| \leq A_{i}, \\
0<\underline{d}_{j} \leq d_{j}(v) \leq \bar{d}_{j}, \quad\left|d_{j}^{\prime}(v)\right| \leq D_{j}, \\
0<\underline{b}_{i} \leq b_{i}^{\prime}(u) \leq \bar{b}_{i}, \quad 0<\underline{e}_{j} \leq e_{j}^{\prime}(v) \leq \bar{e}_{j},
\end{gathered}
$$

for all $u, v \in R$.

$\left(H_{2}\right)$ For each $i=1,2, \ldots, n, j=1,2, \ldots, m$, the activation functions $f_{j}, g_{i}$ satisfy Lipschitz condition, and there exist constants $l_{j}>0, \bar{f}_{j}>0, k_{i}>0$, and $\bar{g}_{i}>0$, such that

$$
\begin{array}{ll}
\left|f_{j}\left(v_{1}\right)-f_{j}\left(v_{2}\right)\right| \leq l_{j}\left|v_{1}-v_{2}\right|, & \left|f_{j}(v)\right| \leq \bar{f}_{j}, \\
& v_{1}, v_{2}, v \in R, \\
\left|g_{i}\left(u_{1}\right)-g_{i}\left(u_{2}\right)\right| \leq k_{i}\left|u_{1}-u_{2}\right|, & \left|g_{i}(u)\right| \leq \bar{g}_{i}, \\
& u_{1}, u_{2}, u \in R,
\end{array}
$$

$\left(H_{3}\right)$ For each $i=1,2, \ldots, n, j=1,2, \ldots, m, I_{i}(t), J_{j}(t)$ are continuously periodic functions defined on $t \in$ $[0, \infty)$ with common period $\omega>0$ and satisfy $0<$ $\underline{I}_{i} \leq I_{i}(t) \leq \bar{I}_{i}, 0<\underline{J}_{j} \leq J_{j}(t) \leq \bar{J}_{j}$.

$\left(H_{4}\right)$ Let $B_{i}\left(u_{i}\right)=a_{i}\left(u_{i}\right) b_{i}\left(u_{i}\right)$; there exist constants $T_{i}>0$ and $K_{i}>0$, such that

$$
0<T_{i} \leq B_{i}^{\prime}(u) \leq K_{i}, \quad i=1,2, \ldots, n, u_{i} \in R .
$$

$\left(H_{5}\right)$ Let $E_{j}\left(v_{j}\right)=d_{j}\left(v_{j}\right) e_{j}\left(v_{j}\right)$; there exist constants $T_{j}^{*}>0$ and $K_{j}^{*}>0$, such that

$0<T_{j}^{*} \leq E_{j}^{\prime}(v) \leq K_{j}^{*}, \quad j=1,2, \ldots, m, v_{j} \in R$. 
$\tau_{i j}(t)$ and $\sigma_{j i}(t)$ are continuously differentiable periodic functions, and there exist constants $0<\bar{\tau}_{i j}<1$ and $0<\bar{\sigma}_{j i}<$ 1 , such that

$$
\tau_{i j}^{\prime}(t) \leq \bar{\tau}_{i j}<1, \quad \sigma_{j i}^{\prime}(t) \leq \bar{\sigma}_{j i}<1,
$$

where $i=1,2, \ldots, n, j=1,2, \ldots, m$, and $\tau_{i j}^{\prime}(t)$ and $\sigma_{j i}^{\prime}(t)$ express the derivative of $\tau_{i j}(t)$ and $\sigma_{j i}(t)$.

Introducing variable transformation

$$
\begin{gathered}
x_{i}(t)=\frac{d u_{i}(t)}{d t}+u_{i}(t), \quad i=1,2, \ldots, n, \\
y_{j}(t)=\frac{d v_{j}(t)}{d t}+v_{j}(t), \quad j=1,2, \ldots, m,
\end{gathered}
$$

then (3) and (4) can be rewritten as

$$
\begin{aligned}
& \frac{d u_{i}(t)}{d t}=-u_{i}(t)+x_{i}(t), \\
& \frac{d x_{i}(t)}{d t}=-\left(1-\alpha_{i}\right) u_{i}(t)-\left(\alpha_{i}-1\right) x_{i}(t)-a_{i}\left(u_{i}(t)\right) \\
& \times\left[b_{i}\left(u_{i}(t)\right)\right. \\
& \left.-\sum_{j=1}^{m} c_{i j} f_{j}\left(v_{j}\left(t-\tau_{i j}(t)\right)\right)-I_{i}(t)\right], \\
& \frac{d v_{j}(t)}{d t}=-v_{j}(t)+y_{j}(t), \\
& \frac{d y_{j}(t)}{d t}=-\left(1-\beta_{j}\right) v_{j}(t)-\left(\beta_{j}-1\right) y_{j}(t)-d_{j}\left(v_{j}(t)\right) \\
& \times\left[e_{j}\left(v_{j}(t)\right)\right. \\
& \left.-\sum_{i=1}^{n} h_{j i} g_{i}\left(u_{i}\left(t-\sigma_{j i}(t)\right)\right)-J_{j}(t)\right], \\
& u_{i}(s)=\varphi_{u i}(s), \quad \frac{d u_{i}(s)}{d t}=\psi_{u i}(s), \quad-\tau \leq s \leq 0, \\
& x_{i}(s)=\varphi_{u i}(s)+\psi_{u i}(s) \doteq \phi_{u i}(s), \quad-\tau \leq s \leq 0, \\
& v_{j}(s)=\varphi_{v j}(s), \quad \frac{d v_{j}(s)}{d t}=\psi_{v j}(s), \quad-\sigma \leq s \leq 0, \\
& y_{j}(s)=\varphi_{v j}(s)+\psi_{v j}(s) \doteq \chi_{v j}(s), \quad-\sigma \leq s \leq 0,
\end{aligned}
$$

Definition 1. Let

$$
\begin{aligned}
& \bar{u}(t)=\left(\bar{u}_{1}(t), \bar{u}_{2}(t), \ldots, \bar{u}_{n}(t)\right)^{T}, \\
& \bar{v}(t)=\left(\bar{v}_{1}(t), \bar{v}_{2}(t), \ldots, \bar{v}_{m}(t)\right)^{T}
\end{aligned}
$$

be an $\omega$-periodic solution of system (3) with initial value

$$
\begin{array}{lll}
\bar{u}_{i}(s)=\bar{\varphi}_{u i}(s), & \frac{d \bar{u}_{i}(s)}{d t}=\bar{\psi}_{u i}(s), & -\tau \leq s \leq 0, \\
\bar{v}_{j}(s)=\bar{\varphi}_{v j}(s), & \frac{d \bar{v}_{j}(s)}{d t}=\bar{\psi}_{v j}(s), & -\sigma \leq s \leq 0,
\end{array}
$$

for every solution

$$
\begin{gathered}
u(t)=\left(u_{1}(t), u_{2}(t), \ldots, u_{n}(t)\right)^{T}, \\
v(t)=\left(v_{1}(t), v_{2}(t), \ldots, v_{m}(t)\right)^{T}
\end{gathered}
$$

of system (3) with any initial value

$$
\begin{aligned}
& u_{i}(s)=\varphi_{u i}(s), \quad \frac{d u_{i}(s)}{d t}=\psi_{u i}(s), \quad-\tau \leq s \leq 0, \\
& v_{j}(s)=\varphi_{v j}(s), \quad \frac{d v_{j}(s)}{d t}=\psi_{v j}(s), \quad-\sigma \leq s \leq 0 .
\end{aligned}
$$

If there exist constants $\delta>0$ and $M>0$, such that

$$
\begin{aligned}
& \sum_{i=1}^{n}\left(u_{i}(t)-\bar{u}_{i}(t)\right)^{2}+\sum_{j=1}^{m}\left(v_{j}(t)-\bar{v}_{j}(t)\right)^{2} \\
& \leq M e^{-\delta t}\left[\left\|\varphi_{u i}-\bar{\varphi}_{u i}\right\|^{2}+\left\|\varphi_{v j}-\bar{\varphi}_{v j}\right\|^{2}\right],
\end{aligned}
$$

for $i=1,2, \ldots, n, j=1,2, \ldots, m$, and $t \geq 0$, then solutions $\bar{u}(t), \bar{v}(t)$ are said to be exponentially stable, where

$$
\begin{gathered}
\left\|\varphi_{u i}-\bar{\varphi}_{u i}\right\|^{2}=\sup _{-\tau \leq t \leq 0} \sum_{i=1}^{n}\left|\varphi_{u i}(t)-\bar{\varphi}_{u i}(t)\right|^{2}, \\
\left\|\varphi_{v j}-\bar{\varphi}_{v j}\right\|^{2}=\sup _{-\sigma \leq t \leq 0} \sum_{j=1}^{m}\left|\varphi_{v j}(t)-\bar{\varphi}_{v j}(t)\right|^{2} .
\end{gathered}
$$

\section{Main Results}

In this section, we can derive some sufficient conditions which ensure the existence and exponential stability of periodic solutions for system (3).

Theorem 2. For system (3), under the hypotheses $\left(H_{1}\right)-\left(H_{3}\right)$, then $u_{i}(t), u_{i}^{\prime}(t), v_{j}(t)$, and $v_{j}^{\prime}(t)$ are bounded, $i=1,2, \ldots, n$, $j=1,2, \ldots, m$, and $t \geq 0$.

Proof. If $u_{i}(t)>0$, then we have

$$
\frac{d\left|u_{i}(t)\right|}{d t}=\frac{d u_{i}(t)}{d t}
$$

if $u_{i}(t)<0$, then

$$
\frac{d\left|u_{i}(t)\right|}{d t}=-\frac{d u_{i}(t)}{d t} .
$$


Hence, $d\left|u_{i}(t)\right| / d t=\operatorname{sgn}\left(u_{i}(t)\right)\left(d u_{i}(t) / d t\right)$. Similarly, we can get

$$
\begin{aligned}
\frac{d\left|v_{i}(t)\right|}{d t} & =\operatorname{sgn}\left(v_{i}(t)\right) \frac{d v_{i}(t)}{d t}, \\
\frac{d^{2}\left|u_{i}(t)\right|}{d t^{2}} & =\operatorname{sgn}\left(u_{i}(t)\right) \frac{d^{2} u_{i}(t)}{d t^{2}}, \\
\frac{d^{2}\left|v_{i}(t)\right|}{d t^{2}} & =\operatorname{sgn}\left(v_{i}(t)\right) \frac{d^{2} v_{i}(t)}{d t^{2}} .
\end{aligned}
$$

Since $b_{i}\left(u_{i}\right)$ are differentiable on $u_{i}(i=1,2, \ldots, n)$, and then we have

$$
b_{i}\left(u_{i}(t)\right)-b_{i}(0)=b_{i}^{\prime}\left(u_{i}^{*}\right) u_{i}(t),
$$

where $u_{i}^{*}$ lies between $u_{i}$ and 0 .

It follows from (3) that

$$
\begin{aligned}
\frac{d^{2}\left|u_{i}(t)\right|}{d t^{2}}= & -\alpha_{i} \frac{d\left|u_{i}(t)\right|}{d t}-\operatorname{sgn}\left(u_{i}(t)\right) a_{i}\left(u_{i}(t)\right) \\
& \times\left[b_{i}\left(u_{i}(t)\right)-\sum_{j=1}^{m} c_{i j} f_{j}\left(v_{j}\left(t-\tau_{i j}(t)\right)\right)+I_{i}(t)\right] \\
= & -\alpha_{i} \frac{d\left|u_{i}(t)\right|}{d t}-\operatorname{sgn}\left(u_{i}(t)\right) a_{i}\left(u_{i}(t)\right) \\
& \times\left[b_{i}\left(u_{i}(t)\right)-b_{i}(0)+b_{i}(0)\right. \\
& \left.-\sum_{j=1}^{m} c_{i j} f_{j}\left(v_{j}\left(t-\tau_{i j}(t)\right)\right)+I_{i}(t)\right] \\
= & -\alpha_{i} \frac{d\left|u_{i}(t)\right|}{d t}-\operatorname{sgn}\left(u_{i}(t)\right) a_{i}\left(u_{i}(t)\right) \\
& \times\left[b_{i}^{\prime}\left(u_{i}^{*}\right) u_{i}(t)+b_{i}(0)\right. \\
& \left.-\sum_{j=1}^{m} c_{i j} f_{j}\left(v_{j}\left(t-\tau_{i j}(t)\right)\right)+I_{i}(t)\right] \\
\leq & -\alpha_{i} \frac{d\left|u_{i}(t)\right|}{d t}-\underline{a}_{i} \underline{b}_{i}\left|u_{i}(t)\right|+\bar{a}_{i} \\
& {\left[\left|b_{i}(0)\right|+\sum_{j=1}^{m}\left|c_{i j}\right| \bar{f}_{j}+\bar{I}_{i}\right] }
\end{aligned}
$$

Similarly, we can obtain

$$
\begin{aligned}
\frac{d^{2}\left|v_{j}(t)\right|}{d t^{2}} \leq & -\beta_{j} \frac{d\left|v_{j}(t)\right|}{d t}-\underline{d}_{j} \underline{e}_{j}\left|v_{j}(t)\right| \\
& +\bar{d}_{j}\left[\left|e_{j}(0)\right|+\sum_{i=1}^{n}\left|h_{j i}\right| \bar{g}_{i}+\bar{J}_{j}\right] .
\end{aligned}
$$

From (21), (22), we can obtain

$$
\left|u_{i}(t)\right| \leq C_{1} e^{\lambda_{1} t}+C_{2} e^{\lambda_{2} t}+\frac{\bar{a}_{i}}{\underline{a}_{i} \underline{b}_{i}}\left[\left|b_{i}(0)\right|+\sum_{j=1}^{m}\left|c_{i j}\right| \bar{f}_{j}+\bar{I}_{i}\right],
$$

where $\lambda_{1,2}=\left(-\alpha_{i} \pm \sqrt{\alpha_{i}^{2}-4 \underline{a}_{i} \underline{b}_{i}}\right) / 2$ and $C_{1}, C_{2}$ are any real constants:

$$
\left|v_{j}(t)\right| \leq C_{1}^{*} e^{\lambda_{1}^{*} t}+C_{2}^{*} e^{\lambda_{2}^{*} t}+\frac{\bar{d}_{j}}{\underline{d}_{j} \underline{e}_{j}}\left[\left|e_{j}(0)\right|+\sum_{i=1}^{n}\left|h_{j i}\right| \bar{g}_{i}+\bar{J}_{j}\right],
$$

where $\lambda_{1,2}^{*}=\left(-\beta_{j} \pm \sqrt{\beta_{j}^{2}-4 \underline{d}_{j} \underline{e}_{j}}\right) / 2$ and $C_{1}^{*}, C_{2}^{*}$ are any real constants.

Since $\alpha_{i}>0, \beta_{j}>0$, we have $\operatorname{Re}\left(\lambda_{1}\right)<0, \operatorname{Re}\left(\lambda_{2}\right)<0$, $\operatorname{Re}\left(\lambda_{1}^{*}\right)<0$, and $\operatorname{Re}\left(\lambda_{2}^{*}\right)<0$, and formula (23) shows that all solutions $u_{i}(t)$ to (3) are bounded for $i=1,2, \ldots, n, t \geq 0$.

Formula (24) shows that all solutions $v_{j}(t)$ to (3) are bounded for $j=1,2, \ldots, m, t \geq 0$.

On the other hand, from (3) we also can obtain

$$
\begin{aligned}
& \frac{d u_{i}(t)}{d t}= e^{-\alpha_{i} t} \frac{d u_{i}(0)}{d t}-e^{-\alpha_{i} t} \int_{0}^{t} e^{\alpha_{i} s} a_{i}\left(u_{i}(s)\right) \\
& \times {\left[b_{i}\left(u_{i}(s)\right)\right.} \\
&\left.-\sum_{j=1}^{m} c_{i j} f_{j}\left(v_{j}\left(s-\tau_{i j}(t)\right)\right)+I_{i}(s)\right] d s, \\
& \frac{d v_{j}(t)}{d t}= e^{-\beta_{j} t} \frac{d v_{j}(0)}{d t}-e^{-\beta_{j} t} \int_{0}^{t} e^{\beta_{i} s} d_{j}\left(v_{j}(s)\right) \\
& \times {\left[e_{j}\left(v_{j}(s)\right)-\sum_{i=1}^{n} h_{j i} g_{i}\right.} \\
&\left.\times\left(u_{i}\left(s-\sigma_{j i}(t)\right)\right)+J_{j}(s)\right] d s, \\
& \quad j=1,2, \ldots, m .
\end{aligned}
$$

Since $u_{i}(t), v_{j}(t)$ are bounded, we may assume that $\left|u_{i}(t)\right| \leq R_{i},\left|v_{j}(t)\right| \leq R_{j}^{*}$, where $R_{i}>0, R_{j}^{*}>0$ are constants, $i=1,2, \ldots, n, j=1,2, \ldots, m$.

From (25), we have

$$
\left|\frac{d u_{i}(t)}{d t}\right| \leq\left|\psi_{u i}(0)\right|+\frac{\bar{a}_{i}}{\alpha_{i}}\left[\bar{b}_{i} R_{i}+\left|b_{i}(0)\right|+\sum_{j=1}^{m}\left|c_{i j}\right| \bar{f}_{j}+\bar{I}_{i}\right] .
$$

Formula (27) shows that all solutions $u_{i}^{\prime}(t)$ are bounded for $i=1,2, \ldots, n, t \geq 0$. 
From (26), we have

$$
\begin{aligned}
& \left|\frac{d v_{j}(t)}{d t}\right| \leq\left|\psi_{v j}(0)\right| \\
& \quad+\frac{\bar{d}_{j}}{\beta_{j}}\left[\bar{e}_{j} R_{j}^{*}+\left|e_{j}(0)\right|+\sum_{i=1}^{n}\left|h_{j i}\right| \bar{g}_{i}+\bar{J}_{j}\right] .
\end{aligned}
$$

Formula (28) shows that all solutions $v_{j}^{\prime}(t)$ are bounded for $j=1,2, \ldots, m, t \geq 0$.

Theorem 3. Under the hypotheses $\left(H_{1}\right)-\left(H_{5}\right)$, if $\alpha_{i}-K_{i}>0$, $\beta_{j}-K_{j}^{*}>0$, and

$$
\begin{gathered}
\alpha_{i}-T_{i}-2+A_{i}\left(\sum_{j=1}^{m}\left|c_{i j}\right| \bar{f}_{j}+\bar{I}_{i}\right)+\sum_{j=1}^{m} \bar{d}_{j} \frac{\left|h_{j i}\right|}{1-\bar{\sigma}_{j i}} k_{i}<0, \\
2-\alpha_{i}-T_{i}+\bar{a}_{i} \sum_{j=1}^{m}\left|c_{i j}\right| l_{j}+A_{i}\left(\sum_{j=1}^{m}\left|c_{i j}\right| \bar{f}_{j}+\bar{I}_{i}\right)<0, \\
\beta_{j}-T_{j}^{*}-2+D_{j}\left(\sum_{i=1}^{n}\left|h_{j i}\right| \bar{g}_{i}+\bar{J}_{j}\right)+\sum_{i=1}^{n} \bar{a}_{i} \frac{\left|c_{i j}\right|}{1-\bar{\tau}_{i j}} l_{j}<0, \\
2-\beta_{j}-T_{j}^{*}+\bar{d}_{j} \sum_{i=1}^{n}\left|h_{j i}\right| k_{i}+D_{j}\left(\sum_{i=1}^{n}\left|h_{j i}\right| \bar{g}_{i}+\bar{J}_{j}\right)<0,
\end{gathered}
$$

for $i=1,2, \ldots, n, j=1,2, \ldots, m$, then system (3) has one $\omega$ periodic solution, which is exponentially stable.

Proof. If $u_{i}^{*}(t), x_{i}^{*}(t), v_{i}^{*}(t)$, and $y_{i}^{*}(t)$ are $\omega$-periodic solution of (11), which are exponentially stable, then we can obtain that $\left(u_{i}^{*}(t), v_{i}^{*}(t)\right)$ are $\omega$-periodic solution of system (3), which is exponentially stable. In the following we only prove that (11) has one $\omega$-periodic solution, which is exponentially stable.

Let

$$
\begin{aligned}
& \bar{u}(t)=\left(\bar{u}_{1}(t), \bar{u}_{2}(t), \ldots, \bar{u}_{n}(t)\right)^{T}, \\
& \bar{v}(t)=\left(\bar{v}_{1}(t), \bar{v}_{2}(t), \ldots, \bar{v}_{m}(t)\right)^{T}
\end{aligned}
$$

be solution of system (3) with initial value (IV1), and let

$$
\begin{gathered}
u(t)=\left(u_{1}(t), u_{2}(t), \ldots, u_{n}(t)\right)^{T} \\
v(t)=\left(v_{1}(t), v_{2}(t), \ldots, v_{m}(t)\right)^{T}
\end{gathered}
$$

be solution of system (3) with any initial value (IV2).

Let

$$
\begin{aligned}
\bar{x}_{i}(t)=\frac{d \bar{u}_{i}(t)}{d t}+\bar{u}_{i}(t), & \bar{y}_{j}(t)=\frac{d \bar{v}_{j}(t)}{d t}+\bar{v}_{j}(t), \\
z_{i}(t)=u_{i}(t)-\bar{u}_{i}(t), & w_{i}(t)=x_{i}(t)-\bar{x}_{i}(t), \\
p_{j}(t)=v_{j}(t)-\bar{v}_{j}(t), & q_{j}(t)=y_{j}(t)-\bar{y}_{j}(t),
\end{aligned}
$$

for $i=1,2, \ldots, n, j=1,2, \ldots, m$.
From (11), we can obtain

$$
\begin{aligned}
& \frac{d z_{i}(t)}{d t}=-z_{i}(t)+w_{i}(t) \\
\frac{d w_{i}(t)}{d t}=- & \left(1-\alpha_{i}\right) z_{i}(t)-\left(\alpha_{i}-1\right) w_{i}(t) \\
+ & a_{i}\left(u_{i}(t)\right)\left[\sum _ { j = 1 } ^ { m } c _ { i j } \left[f_{j}\left(v_{j}\left(t-\tau_{i j}(t)\right)\right)\right.\right. \\
+ & \left(a_{i}\left(u_{i}(t)\right)-a_{i}\left(\bar{u}_{i}(t)\right)\right) \\
& \times\left[\sum_{j=1}^{m} c_{i j} f_{j}\left(\bar{v}_{j}\left(t-\tau_{i j}(t)\right)\right)+I_{i}(t)\right] \\
& \left.-\left[a_{i}\left(u_{i}(t)\right) b_{i}\left(u_{i}(t)\right)-a_{i}\left(\bar{u}_{i}(t)\right)\right)\right]
\end{aligned}
$$

for $i=1,2, \ldots, n$

$$
\begin{aligned}
\frac{d p_{j}(t)}{d t}=-p_{j}(t)+q_{j}(t) \\
\frac{d q_{j}(t)}{d t}=-\left(1-\beta_{j}\right) p_{j}(t)-\left(\beta_{j}-1\right) q_{j}(t) \\
+d_{j}\left(v_{j}(t)\right)\left[\sum _ { i = 1 } ^ { n } h _ { j i } \left[g_{i}\left(u_{i}\left(t-\sigma_{j i}(t)\right)\right)\right.\right. \\
+\left(d_{j}\left(v_{j}(t)\right)-d_{j}\left(\bar{v}_{j}(t)\right)\right) \\
\times\left[\sum_{i=1}^{n} h_{j i} g_{i}\left(\bar{u}_{i}\left(t-\sigma_{j i}(t)\right)\right)+J_{j}(t)\right] \\
\left.\left.\left.-\left[d_{j}\left(v_{j}(t)\right) e_{j}\left(v_{j}(t)\right)-\sigma_{j i}(t)\right)\right)\right]\right]
\end{aligned}
$$

for $j=1,2, \ldots, m$.

Since functions $a_{i}(u)$ and $b_{i}(u)$ are differentiable, using differential mean value theorem, we have

$$
\begin{gathered}
a_{i}\left(u_{i}(t)\right)-a_{i}\left(\bar{u}_{i}(t)\right)=a_{i}^{\prime}\left(\xi_{i}\right) z_{i}(t), \\
a_{i}\left(u_{i}(t)\right) b_{i}\left(u_{i}(t)\right)-a_{i}\left(\bar{u}_{i}(t)\right) b_{i}\left(\bar{u}_{i}(t)\right) \\
=B_{i}\left(u_{i}\right)-B_{i}\left(\bar{u}_{i}\right)=B_{i}^{\prime}\left(\bar{\xi}_{i}\right) z_{i}(t),
\end{gathered}
$$

where $\xi_{i}$ and $\bar{\xi}_{i}$ lie between $u_{i}$ and $\bar{u}_{i}$.

Since $0<T_{i} \leq B_{i}^{\prime}(u) \leq K_{i}$, if $\alpha_{i}-K_{i}>0$, then we have $\alpha_{i}-B_{i}^{\prime}\left(\xi_{i}\right) \geq \alpha_{i}-K_{i}>0$ and $0<\alpha_{i}-B_{i}^{\prime}\left(\xi_{i}\right) \leq \alpha_{i}-T_{i}$. 
From (33) we get

$$
\begin{aligned}
& \frac{1}{2} \frac{d z_{i}^{2}(t)}{d t}=-z_{i}^{2}(t)+z_{i}(t) w_{i}(t), \\
& \frac{1}{2} \frac{d w_{i}^{2}(t)}{d t}=-\left(1-\alpha_{i}\right) z_{i}(t) w_{i}(t)-\left(\alpha_{i}-1\right) w_{i}^{2}(t) \\
&+a_{i}\left(u_{i}(t)\right) w_{i}(t) \\
& \times\left[\sum _ { j = 1 } ^ { m } c _ { i j } \left[f_{j}\left(v_{j}\left(t-\tau_{i j}(t)\right)\right)\right.\right. \\
&+\left(a_{i}\left(u_{i}(t)\right)-a_{i}\left(\bar{u}_{i}(t)\right)\right) w_{i}(t) \\
& \cdot\left[\sum_{j=1}^{m} c_{i j} f_{j}\left(\bar{v}_{j}\left(t-\tau_{i j}(t)\right)\right)+I_{i}(t)\right] \\
&-\left[a_{i}\left(u_{i}(t)\right) b_{i}\left(u_{i}(t)\right)\right. \\
&\left.-a_{i}\left(\bar{u}_{i}(t)\right) b_{i}\left(\bar{u}_{i}(t)\right)\right] w_{i}(t) \\
&=-\left(1-\alpha_{i}\right) z_{i}(t) w_{i}(t)-\left(\alpha_{i}-1\right) w_{i}^{2}(t) \\
&+a_{i}\left(u_{i}(t)\right) w_{i}(t) \\
&+B_{i}^{\prime}\left(\bar{\xi}_{i}\right) z_{i}(t) w_{i}(t), \quad i=1,2, \ldots, n . \\
&+\left[\sum _ { j = 1 } ^ { m } c _ { i j } \left[f_{j}\left(v_{j}\left(t-\tau_{i j}(t)\right)\right)\right.\right. \\
& {\left.\left[\sum_{i}\right)\right) z_{i}(t) w_{i}(t) } \\
&\left.\left.-f_{j}\left(\bar{v}_{j}\left(t-\tau_{i j}(t)\right)\right)\right]\right] \\
& \\
& \\
&
\end{aligned}
$$

From (36), we can obtain

$$
\begin{aligned}
& \frac{1}{2} \frac{d}{d t}\left(z_{i}^{2}(t)+w_{i}^{2}(t)\right) \\
& \leq-z_{i}^{2}(t)+\left[\alpha_{i}-B_{i}^{\prime}\left(\bar{\xi}_{i}\right)\right] z_{i}(t) w_{i}(t)-\left(\alpha_{i}-1\right) w_{i}^{2}(t) \\
& \quad+\bar{a}_{i}\left|w_{i}(t)\right| \sum_{j=1}^{m}\left|c_{i j}\right| l_{j}\left|p_{j}\left(t-\tau_{i j}(t)\right)\right| \\
& \quad+A_{i}\left|z_{i}(t)\right|\left|w_{i}(t)\right|\left[\sum_{j=1}^{m}\left|c_{i j}\right| \bar{f}_{j}+\bar{I}_{i}\right]
\end{aligned}
$$

$$
\begin{aligned}
\leq & -z_{i}^{2}(t)+\left[\alpha_{i}-B_{i}^{\prime}\left(\bar{\xi}_{i}\right)\right] \frac{\left(z_{i}^{2}(t)+w_{i}^{2}(t)\right)}{2} \\
& -\left(\alpha_{i}-1\right) w_{i}^{2}(t) \\
& +\bar{a}_{i} \sum_{j=1}^{m}\left|c_{i j}\right| l_{j} \frac{\left(w_{i}^{2}(t)+p_{j}^{2}\left(t-\tau_{i j}(t)\right)\right)}{2} \\
& +A_{i}\left[\sum_{j=1}^{m}\left|c_{i j}\right| \bar{f}_{j}+\bar{I}_{i}\right] \frac{\left(z_{i}^{2}(t)+w_{i}^{2}(t)\right)}{2} \\
= & \frac{1}{2}\left[\alpha_{i}-T_{i}\left(\bar{\xi}_{i}\right)-2+A_{i}\left(\sum_{j=1}^{m}\left|c_{i j}\right| \bar{f}_{j}+\bar{I}_{i}\right)\right] z_{i}^{2}(t) \\
& +\frac{1}{2}\left[2-\alpha_{i}-T_{i}\left(\bar{\xi}_{i}\right)+\bar{a}_{i} \sum_{j=1}^{m}\left|c_{i j}\right| l_{j}\right. \\
& \left.+A_{i}\left(\sum_{j=1}^{m}\left|c_{i j}\right| \bar{f}_{j}+\bar{I}_{i}\right)\right] w_{i}^{2}(t) \\
+ & \frac{\bar{a}_{i}}{2} \sum_{j=1}^{m}\left|c_{i j}\right| l_{j} p_{j}^{2}\left(t-\tau_{i j}(t)\right)
\end{aligned}
$$

for $i=1,2, \ldots, n, t>0$.

Similar to the above derivation, from (34) we can get

$$
\begin{aligned}
& \frac{1}{2} \frac{d}{d t}\left(p_{j}^{2}(t)+q_{j}^{2}(t)\right) \\
& \leq \frac{1}{2}\left[\beta_{j}-T_{j}^{*}\left(\bar{\xi}_{j}\right)-2+D_{j}\left(\sum_{i=1}^{n}\left|h_{j i}\right| \bar{g}_{i}+\bar{J}_{j}\right)\right] p_{j}^{2}(t) \\
& +\frac{1}{2}\left[2-\beta_{j}-T_{j}^{*}\left(\bar{\xi}_{j}\right)+\bar{d}_{j} \sum_{i=1}^{n}\left|h_{j i}\right| k_{i}\right. \\
& \left.\quad+D_{j}\left(\sum_{i=1}^{n}\left|h_{j i}\right| \bar{g}_{i}+\bar{J}_{j}\right)\right] q_{j}^{2}(t) \\
& +\frac{\bar{d}_{j}}{2} \sum_{i=1}^{n}\left|h_{j i}\right| k_{i} z_{i}^{2}\left(t-\sigma_{j i}(t)\right)
\end{aligned}
$$

for $j=1,2, \ldots, m, t>0$.

We consider the Lyapunov functional

$$
\begin{aligned}
V(t)=\sum_{i=1}^{n}\{ & \frac{z_{i}^{2}(t)+w_{i}^{2}(t)}{2} e^{\varepsilon t} \\
& \left.+\frac{\bar{a}_{i}}{2} \sum_{j=1}^{m} \frac{\left|c_{i j}\right|}{1-\bar{\tau}_{i j}} l_{j} \int_{t-\tau_{i j}(t)}^{t} e^{\varepsilon\left(s+\tau_{i j}(s)\right)} p_{j}^{2}(s) d s\right\}
\end{aligned}
$$




$$
\begin{aligned}
+\sum_{j=1}^{m}\left\{\frac{p_{j}^{2}(t)+q_{j}^{2}(t)}{2} e^{\varepsilon t}\right. & \\
& \left.+\frac{\bar{d}_{j}}{2} \sum_{i=1}^{n} \frac{\left|h_{j i}\right|}{1-\bar{\sigma}_{j i}} k_{i} \int_{t-\sigma_{j i}(t)}^{t} e^{\varepsilon\left(s+\sigma_{j i}(s)\right)} z_{i}^{2}(s) d s\right\},
\end{aligned}
$$

where $\varepsilon>0$ is a small number.

Calculating the upper right Dini-derivative $D^{+} V(t)$ of $V(t)$ along the solution of (33) and (34), using (37) and (38), we have

$D^{+} V(t)$

$$
\begin{aligned}
& =\sum_{i=1}^{n}\left\{\varepsilon \frac{z_{i}^{2}(t)+w_{i}^{2}(t)}{2} e^{\varepsilon t}+\frac{1}{2} \frac{d}{d t}\left(z_{i}^{2}(t)+w_{i}^{2}(t)\right) e^{\varepsilon t}\right. \\
& +\frac{\bar{a}_{i}}{2} \sum_{j=1}^{m} \frac{\left|c_{i j}\right|}{1-\bar{\tau}_{i j}} l_{j}\left[p_{j}^{2}(t) e^{\varepsilon\left(t+\tau_{i j}(t)\right)}\right. \\
& \left.\left.-\left(1-\tau_{i j}^{\prime}(t)\right) p_{j}^{2}\left(t-\tau_{i j}(t)\right) e^{\varepsilon t}\right]\right\} \\
& +\sum_{j=1}^{m}\left\{\varepsilon \frac{p_{j}^{2}(t)+q_{j}^{2}(t)}{2} e^{\varepsilon t}+\frac{1}{2} \frac{d}{d t}\left(p_{j}^{2}(t)+q_{j}^{2}(t)\right) e^{\varepsilon t}\right. \\
& +\frac{\bar{d}_{j}}{2} \sum_{i=1}^{n} \frac{\left|h_{j i}\right|}{1-\bar{\sigma}_{j i}} k_{i} \\
& \times\left[z_{i}^{2}(t) e^{\varepsilon\left(t+\sigma_{j i}(t)\right)}-\left(1-\sigma_{j i}^{\prime}(t)\right) z_{i}^{2}\right. \\
& \left.\left.\times\left(t-\sigma_{j i}(t)\right) e^{\varepsilon t}\right]\right\}
\end{aligned}
$$$$
\leq e^{\varepsilon t} \sum_{i=1}^{n}\left\{\varepsilon \frac{z_{i}^{2}(t)+w_{i}^{2}(t)}{2}\right.
$$$$
+\frac{1}{2}\left[\alpha_{i}-T_{i}-2+A_{i}\left(\sum_{j=1}^{m}\left|c_{i j}\right| \bar{f}_{j}+\bar{I}_{i}\right)\right] z_{i}^{2}(t)
$$$$
+\frac{1}{2}\left[2-\alpha_{i}-T_{i}+\bar{a}_{i} \sum_{j=1}^{m}\left|c_{i j}\right| l_{j}\right.
$$$$
\left.+A_{i}\left(\sum_{j=1}^{m}\left|c_{i j}\right| \bar{f}_{j}+\bar{I}_{i}\right)\right] w_{i}^{2}(t)
$$$$
+\frac{\bar{a}_{i}}{2} \sum_{j=1}^{m}\left|c_{i j}\right| l_{j} p_{j}^{2}\left(t-\tau_{i j}(t)\right)+\frac{\bar{a}_{i}}{2} \sum_{j=1}^{m} \frac{\left|c_{i j}\right|}{1-\bar{\tau}_{i j}} l_{j}
$$$$
\left.\times\left[p_{j}^{2}(t) e^{\varepsilon \tau}-\left(1-\tau_{i j}^{\prime}(t)\right) p_{j}^{2}\left(t-\tau_{i j}(t)\right)\right]\right\}
$$$$
+e^{\varepsilon t} \sum_{j=1}^{m}\left\{\varepsilon \frac{p_{j}^{2}(t)+q_{j}^{2}(t)}{2}\right.
$$$$
+\frac{1}{2}\left[\beta_{j}-T_{j}^{*}-2\right.
$$$$
\left.+D_{j}\left(\sum_{i=1}^{n}\left|h_{j i}\right| \bar{g}_{i}+\bar{J}_{j}\right)\right] p_{j}^{2}(t)
$$$$
+\frac{1}{2}\left[2-\beta_{j}-T_{j}^{*}+\bar{d}_{j} \sum_{i=1}^{n}\left|h_{j i}\right| k_{i}\right.
$$$$
\left.+D_{j}\left(\sum_{i=1}^{n}\left|h_{j i}\right| \bar{g}_{i}+\bar{J}_{j}\right)\right] q_{j}^{2}(t)
$$$$
+\frac{\bar{d}_{j}}{2} \sum_{i=1}^{n}\left|h_{j i}\right| k_{i} z_{i}^{2}\left(t-\sigma_{j i}(t)\right)
$$$$
+\frac{\bar{d}_{j}}{2} \sum_{i=1}^{n} \frac{\left|h_{j i}\right|}{1-\bar{\sigma}_{j i}} k_{i}
$$$$
\left.\times\left[z_{i}^{2}(t) e^{\varepsilon \sigma}-\left(1-\sigma_{j i}^{\prime}(t)\right) z_{i}^{2}\left(t-\sigma_{j i}(t)\right)\right]\right\}
$$$$
\leq e^{\varepsilon t} \sum_{i=1}^{n}\left\{\frac { 1 } { 2 } \left[\varepsilon+\alpha_{i}-T_{i}-2+A_{i}\left(\sum_{j=1}^{m}\left|c_{i j}\right| \bar{f}_{j}+\bar{I}_{i}\right)\right.\right.
$$$$
\left.+\sum_{j=1}^{m} \bar{d}_{j} \frac{\left|h_{j i}\right|}{1-\bar{\sigma}_{j i}} k_{i} e^{\varepsilon \sigma}\right] z_{i}^{2}(t)
$$$$
+\frac{1}{2}\left[\varepsilon+2-\alpha_{i}-T_{i}+\bar{a}_{i} \sum_{j=1}^{m}\left|c_{i j}\right| l_{j}\right.
$$$$
\left.\left.+A_{i}\left(\sum_{j=1}^{m}\left|c_{i j}\right| \bar{f}_{j}+\bar{I}_{i}\right)\right] w_{i}^{2}(t)\right\}
$$$$
+e^{\varepsilon t} \sum_{j=1}^{m}\left\{\frac { 1 } { 2 } \left[\varepsilon+\beta_{j}-T_{j}^{*}-2+D_{j}\left(\sum_{i=1}^{n}\left|h_{j i}\right| \bar{g}_{i}+\bar{J}_{j}\right)\right.\right.
$$$$
\left.+\sum_{i=1}^{n} \bar{a}_{i} \frac{\left|c_{i j}\right|}{1-\bar{\tau}_{i j}} l_{j} e^{\varepsilon \tau}\right] p_{j}^{2}(t)
$$

$$
\begin{aligned}
& +\frac{1}{2}\left[\varepsilon+2-\beta_{j}-T_{j}^{*}+\bar{d}_{j} \sum_{i=1}^{n}\left|h_{j i}\right| k_{i}\right. \\
& \left.\left.+D_{j}\left(\sum_{i=1}^{n}\left|h_{j i}\right| \bar{g}_{i}+\bar{J}_{j}\right)\right] q_{j}^{2}(t)\right\} .
\end{aligned}
$$


From condition of Theorem 3, we can choose a small $\varepsilon>0$ such that

$\varepsilon+\alpha_{i}-T_{i}-2+A_{i}\left(\sum_{j=1}^{m}\left|c_{i j}\right| \bar{f}_{j}+\bar{I}_{i}\right)+\sum_{j=1}^{m} \bar{d}_{j} \frac{\left|h_{j i}\right|}{1-\bar{\sigma}_{j i}} k_{i} e^{\varepsilon \sigma}$

$\leq 0$,

$\varepsilon+2-\alpha_{i}-T_{i}+\bar{a}_{i} \sum_{j=1}^{m}\left|c_{i j}\right| l_{j}+A_{i}\left(\sum_{j=1}^{m}\left|c_{i j}\right| \bar{f}_{j}+\bar{I}_{i}\right)$

$\leq 0$,

$\varepsilon+\beta_{j}-T_{j}^{*}-2+D_{j}\left(\sum_{i=1}^{n}\left|h_{j i}\right| \bar{g}_{i}+\bar{J}_{j}\right)+\sum_{i=1}^{n} \bar{a}_{i} \frac{\left|c_{i j}\right|}{1-\bar{\tau}_{i j}} l_{j} e^{\varepsilon \tau}$ $\leq 0$,

$\varepsilon+2-\beta_{j}-T_{j}^{*}+\bar{d}_{j} \sum_{i=1}^{n}\left|h_{j i}\right| k_{i}+D_{j}\left(\sum_{i=1}^{n}\left|h_{j i}\right| \bar{g}_{i}+\bar{J}_{j}\right) \leq 0$,

From (40), we get $D^{+} V(t) \leq 0$ and so $V(t) \leq V(0)$, for all $t \geq 0$. From (39), we have

$$
\begin{gathered}
V(t) \geq \sum_{i=1}^{n} \frac{z_{i}^{2}(t)+w_{i}^{2}(t)}{2} e^{\varepsilon t}+\sum_{j=1}^{m} \frac{p_{j}^{2}(t)+q_{j}^{2}(t)}{2} e^{\varepsilon t} \\
=\sum_{i=1}^{n} \frac{e^{\varepsilon t}}{2}\left[\left(u_{i}(t)-\bar{u}_{i}(t)\right)^{2}+\left(x_{i}(t)-\bar{x}_{i}(t)\right)^{2}\right] \\
+\sum_{j=1}^{m} \frac{e^{\varepsilon t}}{2}\left[\left(v_{j}(t)-\bar{v}_{j}(t)\right)^{2}+\left(y_{j}(t)-\bar{y}_{j}(t)\right)^{2}\right], \\
V(0)=\sum_{i=1}^{n}\left\{\frac{z_{i}^{2}(0)+w_{i}^{2}(0)}{2}\right. \\
\left.+\frac{\bar{a}_{i}}{2} \sum_{j=1}^{m} \frac{\left|c_{i j}\right|}{1-\bar{\tau}_{i j}} l_{j} \int_{-\tau_{i j}(t)}^{0} e^{\varepsilon\left(s+\tau_{i j}(s)\right)} p_{j}^{2}(s) d s\right\} \\
+\sum_{j=1}^{m}\left\{\begin{array}{c}
\frac{p_{j}^{2}(0)+q_{j}^{2}(0)}{2} \\
\quad \bar{d}_{j} \sum_{i=1}^{n} \frac{\left|h_{j i}\right|}{1-\bar{\sigma}_{j i}} k_{i} \\
\left.\times \int_{-\sigma_{j i}(t)}^{0} e^{\varepsilon\left(s+\sigma_{j i}(s)\right)} z_{i}^{2}(s) d s\right\}
\end{array}\right.
\end{gathered}
$$$$
=\sum_{i=1}^{n}\left\{\frac{\left(\varphi_{u i}(0)-\bar{\varphi}_{u i}(0)\right)^{2}}{2}+\frac{\left(\phi_{u i}(0)-\bar{\phi}_{u i}(0)\right)^{2}}{2}\right.
$$$$
+\frac{\bar{a}_{i}}{2} \sum_{j=1}^{m} \frac{\left|c_{i j}\right|}{1-\bar{\tau}_{i j}} l_{j}
$$$$
\left.\times \int_{-\tau_{i j}(t)}^{0} e^{\varepsilon\left(s+\tau_{i j}(s)\right)}\left(\varphi_{v j}(s)-\bar{\varphi}_{v j}(s)\right)^{2} d s\right\}
$$$$
+\sum_{j=1}^{m}\left\{\frac{\left(\varphi_{v j}(0)-\bar{\varphi}_{v j}(0)\right)^{2}}{2}+\frac{\left(\chi_{v j}(0)-\bar{\chi}_{v j}(0)\right)^{2}}{2}\right.
$$$$
+\frac{\bar{d}_{j}}{2} \sum_{i=1}^{n} \frac{\left|h_{j i}\right|}{1-\bar{\sigma}_{j i}} k_{i}
$$$$
\left.\times \int_{-\sigma_{j i}(s)}^{0} e^{\varepsilon\left(s+\sigma_{j i}(t)\right)}\left(\varphi_{u i}(s)-\bar{\varphi}_{u i}(s)\right)^{2} d s\right\}
$$$$
\leq \frac{\left\|\varphi_{u i}-\bar{\varphi}_{u i}\right\|^{2}}{2}+\frac{\left\|\phi_{u i}-\bar{\phi}_{u i}\right\|^{2}}{2}
$$$$
+\tau \sum_{i=1}^{n} \frac{\bar{a}_{i}}{2} \max _{1 \leq j \leq m}\left\{\frac{\left|c_{i j}\right|}{1-\bar{\tau}_{i j}} l_{j}\right\} e^{\varepsilon \tau}\left\|\varphi_{v j}-\bar{\varphi}_{v j}\right\|^{2}
$$$$
+\frac{\left\|\varphi_{v j}-\bar{\varphi}_{v j}\right\|^{2}}{2}+\frac{\left\|\chi_{v j}-\bar{\chi}_{v j}\right\|^{2}}{2}
$$$$
+\sigma \sum_{j=1}^{m} \frac{\bar{d}_{j}}{2} \max _{1 \leq i \leq n}\left\{\frac{\left|h_{j i}\right|}{1-\bar{\sigma}_{j i}} k_{i}\right\} e^{\varepsilon \sigma}\left\|\varphi_{u i}-\bar{\varphi}_{u i}\right\|^{2}
$$$$
=\left[1+\sigma \sum_{j=1}^{m} \bar{d}_{j_{1 \leq i \leq n}}\left\{\frac{\left|h_{j i}\right|}{1-\bar{\sigma}_{j i}} k_{i}\right\} e^{\varepsilon \sigma}\right] \frac{\left\|\varphi_{u i}-\bar{\varphi}_{u i}\right\|^{2}}{2}
$$$$
+\left[1+\tau \sum_{i=1}^{n} \bar{a}_{i} \max _{1 \leq j \leq m}\left\{\frac{\left|c_{i j}\right|}{1-\bar{\tau}_{i j}} l_{j}\right\} e^{\varepsilon \tau}\right] \frac{\left\|\varphi_{v j}-\bar{\varphi}_{v j}\right\|^{2}}{2}
$$$$
+\frac{\left\|\phi_{u i}-\bar{\phi}_{u i}\right\|^{2}}{2}+\frac{\left\|\chi_{v j}-\bar{\chi}_{v j}\right\|^{2}}{2}
$$$$
\leq M^{*}\left[\frac{\left\|\varphi_{u i}-\bar{\varphi}_{u i}\right\|^{2}}{2}+\frac{\left\|\varphi_{v j}-\bar{\varphi}_{v j}\right\|^{2}}{2}\right]
$$$$
+\frac{\left\|\phi_{u i}-\bar{\phi}_{u i}\right\|^{2}}{2}+\frac{\left\|\chi_{v j}-\bar{\chi}_{v j}\right\|^{2}}{2}
$$$$
=\frac{M}{2}\left[\left\|\varphi_{u i}-\bar{\varphi}_{u i}\right\|^{2}+\left\|\varphi_{v j}-\bar{\varphi}_{v j}\right\|^{2}\right] \text {, }
$$ 
where $M^{*}=\max \left\{1+\sigma \sum_{j=1}^{m} \bar{d}_{j} \max _{1 \leq i \leq n}\left\{\left(\left|h_{j i}\right| /(1-\right.\right.\right.$ $\left.\left.\left.\left.\bar{\sigma}_{j i}\right)\right) k_{i}\right\} e^{\varepsilon \sigma}, 1+\tau \sum_{i=1}^{n} \bar{a}_{i} \max _{1 \leq j \leq m}\left\{\left(\left|c_{i j}\right| /\left(1-\bar{\tau}_{i j}\right)\right) l_{j}\right\} e^{\varepsilon \tau}\right\}$,

$$
M=M^{*}+\frac{\left\|\phi_{u i}-\bar{\phi}_{u i}\right\|^{2}+\left\|\chi_{v j}-\bar{\chi}_{v j}\right\|^{2}}{\left\|\varphi_{u i}-\bar{\varphi}_{u i}\right\|^{2}+\left\|\varphi_{v j}-\bar{\varphi}_{v j}\right\|^{2}} .
$$

Since $V(0) \geq V(t)$, from (42), we obtain

$$
\begin{aligned}
& \sum_{i=1}^{n} {\left[\left(u_{i}(t)-\bar{u}_{i}(t)\right)^{2}+\left(x_{i}(t)-\bar{x}_{i}(t)\right)^{2}\right] } \\
&+\sum_{j=1}^{m}\left[\left(v_{j}(t)-\bar{v}_{j}(t)\right)^{2}+\left(y_{j}(t)-\bar{y}_{j}(t)\right)^{2}\right] \\
&<M e^{-\varepsilon t}\left[\left\|\varphi_{u i}-\bar{\varphi}_{u i}\right\|^{2}+\left\|\varphi_{v j}-\bar{\varphi}_{v j}\right\|^{2}\right] .
\end{aligned}
$$

From (44), we obtain

$$
\begin{gathered}
\sum_{i=1}^{n}\left(u_{i}(t)-\bar{u}_{i}(t)\right)^{2}+\sum_{j=1}^{m}\left(v_{j}(t)-\bar{v}_{j}(t)\right)^{2} \\
<M e^{-\varepsilon t}\left[\left\|\varphi_{u i}-\bar{\varphi}_{u i}\right\|^{2}+\left\|\varphi_{v j}-\bar{\varphi}_{v j}\right\|^{2}\right] .
\end{gathered}
$$

For $i=1,2, \ldots, n, j=1,2, \ldots, m$, when $I_{i}(t), J_{j}(t)$, $\tau_{i j}(t)$, and $\sigma_{j i}$ are continuously periodic functions defined on $t \in[0, \infty)$ with common period $\omega>0$, if $u_{i}(t), v_{j}(t)$ are the solutions of (3), then for any natural number $k, u_{i}(t+k \omega)$, $v_{j}(t+k \omega)$ are the solutions of (3). Thus, from (45), there exist constants $N>0$ and $\delta>0$, such that

$$
\begin{aligned}
& \left|u_{i}(t+(k+1) \omega)-u_{i}(t+k \omega)\right| \leq N e^{-\delta(t+k \omega)}, \\
& \left|v_{j}(t+(k+1) \omega)-v_{j}(t+k \omega)\right| \leq N e^{-\delta(t+k \omega)},
\end{aligned}
$$

for $i=1,2, \ldots, n, j=1,2, \ldots, m, t>0$.

It is noted that, for any natural number $p$,

$$
\begin{aligned}
u_{i}(t+(p+1) \omega)= & u_{i}(t) \\
& +\sum_{k=0}^{p}\left(u_{i}(t+(k+1) \omega)-u_{i}(t+k \omega)\right) .
\end{aligned}
$$

Thus

$$
\begin{aligned}
\left|u_{i}(t+(p+1) \omega)\right| \leq & \left|u_{i}(t)\right| \\
& +\sum_{k=0}^{p}\left|u_{i}(t+(k+1) \omega)-u_{i}(t+k \omega)\right|
\end{aligned}
$$

Since $u_{i}(t)$ is bounded, it follows from (46) and (49) that $\{u(t+p \omega)\}$ uniformly converges to a continuous functions $u^{*}(t)=\left(u_{1}^{*}(t), u_{2}^{*}(t), \ldots, u_{n}^{*}(t)\right)$ on any compact set of $R$.

Similarly, since $v_{j}(t)$ is bounded, from $(47),\{v(t+p \omega)\}$ uniformly converges to a continuous function $v^{*}(t)=\left(v_{1}^{*}(t)\right.$, $\left.v_{2}^{*}(t), \ldots, v_{n}^{*}(t)\right)$ on any compact set of $R$.
When $u_{i}(t), u_{i}^{\prime}(t), v_{j}(t)$, and $v_{j}^{\prime}(t)$ are bounded, $x_{i}(t)=$ $u_{i}(t)+u_{i}^{\prime}(t), y_{j}(t)=v_{j}(t)+v_{j}^{\prime}(t)$, and we can obtain that $x_{i}(t), y_{j}(t)$ are bounded. Similarly, from (44), they can be proved that $\{x(t+p \omega)\},\{y(t+p \omega)\}$ uniformly converge to continuous functions $x^{*}(t)=\left(x_{1}^{*}(t), x_{2}^{*}(t), \ldots, x_{n}^{*}(t)\right)$ and $y^{*}(t)=\left(y_{1}^{*}(t), y_{2}^{*}(t), \ldots, y_{m}^{*}(t)\right)$ on any compact set of $R$, respectively.

Now we will show that $\left(u^{* T}(t), v^{* T}(t)\right)^{T}$ is the $\omega$-periodic solution of system (3).

First, $u^{*}(t), v^{*}(t)$ are $\omega$-periodic functions, since

$$
\begin{aligned}
& u^{*}(t+\omega)=\lim _{p \rightarrow \infty} u(t+(p+1) \omega)=u^{*}(t), \\
& v^{*}(t+\omega)=\lim _{p \rightarrow \infty} v(t+(p+1) \omega)=v^{*}(t) .
\end{aligned}
$$

Second, we prove that $\left(u^{* T}(t), v^{* T}(t)\right)^{T}$ is a solution of system (3).

In fact, $I_{i}(t+p \omega)=I_{i}(t), J_{j}(t+p \omega)=J_{j}(t), \tau_{i j}(t+p \omega)=$ $\tau_{i j}(t), \sigma_{j i}(t+p \omega)=\sigma_{j i}(t)$, and

$$
\frac{d u_{i}(t+p \omega)}{d t}=-u_{i}(t+p \omega)+x_{i}(t+p \omega),
$$

$$
\begin{aligned}
& \frac{d x_{i}(t+p \omega)}{d t} \\
& =-\left(1-\alpha_{i}\right) u_{i}(t+p \omega)-\left(\alpha_{i}-1\right) x_{i}(t+p \omega) \\
& -a_{i}\left(u_{i}(t+p \omega)\right) \\
& \times\left[b_{i}\left(u_{i}(t+p \omega)\right)\right. \\
& \left.-\sum_{j=1}^{m} c_{i j} f_{j}\left(v_{j}\left(t+p \omega-\tau_{i j}(t)\right)\right)-I_{i}(t)\right], \\
& \frac{d v_{j}(t+p \omega)}{d t}=-v_{j}(t+p \omega)+y_{j}(t+p \omega), \\
& \frac{d y_{j}(t+p \omega)}{d t} \\
& =-\left(1-\beta_{j}\right) v_{j}(t+p \omega)-\left(\beta_{j}-1\right) y_{j}(t+p \omega) \\
& -d_{j}\left(v_{j}(t+p \omega)\right) \\
& \times\left[e_{j}\left(v_{j}(t+p \omega)\right)\right. \\
& \left.-\sum_{i=1}^{n} h_{j i} g_{i}\left(u_{i}\left(t+p \omega-\sigma_{j i}(t)\right)\right)-J_{j}(t)\right] \text {, }
\end{aligned}
$$

for $i=1,2, \ldots, n, j=1,2, \ldots, m$. 
Since $\{u(t+p \omega)\}$ and $\{v(t+p \omega)\}$ uniformly converge to continuous function

$$
\begin{aligned}
& u^{*}(t)=\left(u_{1}^{*}(t), u_{2}^{*}(t), \ldots, u_{n}^{*}(t)\right), \\
& v^{*}(t)=\left(v_{1}^{*}(t), v_{2}^{*}(t), \ldots, v_{m}^{*}(t)\right),
\end{aligned}
$$

respectively, $\{x(t+p \omega)\}$ and $\{y(t+p \omega)\}$ uniformly converge to a continuous function

$$
\begin{aligned}
& x^{*}(t)=\left(x_{1}^{*}(t), x_{2}^{*}(t), \ldots, x_{n}^{*}(t)\right), \\
& y^{*}(t)=\left(y_{1}^{*}(t), y_{2}^{*}(t), \ldots, y_{m}^{*}(t)\right),
\end{aligned}
$$

respectively. (51) implies that

$$
\begin{array}{lll}
\left\{\frac{d u_{i}(t+p \omega)}{d t}\right\}, & \left\{\frac{d x_{i}(t+p \omega)}{d t}\right\}, \\
\left\{\frac{d v_{j}(t+p \omega)}{d t}\right\}, & \left\{\frac{d y_{j}(t+p \omega)}{d t}\right\}
\end{array}
$$

uniformly converge to continuous functions on any compact set of $R$, respectively. Thus, let $p \rightarrow \infty$; we obtain

$$
\begin{gathered}
\frac{d u_{i}^{*}(t)}{d t}=-u_{i}^{*}(t)+x_{i}^{*}(t), \\
\frac{d x_{i}^{*}(t)}{d t} \\
=-\left(1-\alpha_{i}\right) u_{i}^{*}(t)-\left(\alpha_{i}-1\right) x_{i}^{*}(t) \\
-a_{i}\left(u_{i}^{*}(t)\right) \\
\times\left[b_{i}\left(u_{i}^{*}(t)\right)\right. \\
\frac{\left.-\sum_{j=1}^{m} c_{i j} f_{j}\left(v_{j}^{*}\left(t-\tau_{i j}(t)\right)\right)-I_{i}(t)\right],}{d t}=-v_{j}^{*}(t)+y_{j}^{*}(t),
\end{gathered}
$$$$
\frac{d y_{j}^{*}(t)}{d t}
$$$$
=-\left(1-\beta_{j}\right) v_{j}^{*}(t)-\left(\beta_{j}-1\right) y_{j}^{*}(t)
$$$$
-d_{j}\left(v_{j}^{*}(t)\right)
$$$$
\times\left[e_{j}\left(v_{j}^{*}(t)\right)\right.
$$$$
\left.-\sum_{i=1}^{n} h_{j i} g_{i}\left(u_{i}^{*}\left(t-\sigma_{j i}(t)\right)\right)-J_{j}(t)\right],
$$

for $i=1,2, \ldots, n, j=1,2, \ldots, m$.
Thus, $\left(u^{* T}(t), v^{* T}(t)\right)$ is a periodic solution of system (3). From (45), we obtain that system (3) has one $\omega$-periodic solution, which is exponentially stable.

Theorem 4. Under the hypotheses $\left(H_{1}\right)-\left(H_{5}\right)$, there is $\omega$ periodic solution of system (3), which is exponentially stable, if the following conditions hold:

$$
\begin{gathered}
\alpha_{i}-1-T_{i}>0, \quad \beta_{j}-1-T_{j}^{*}>0, \\
-T_{i}+A_{i} \sum_{j=1}^{m} \bar{f}_{j}\left|c_{i j}\right|+A_{i} \bar{I}_{i}+\bar{a}_{i} \sum_{j=1}^{m} l_{j}\left|c_{i j}\right|<0, \\
-T_{j}^{*}+D_{j} \sum_{i=1}^{n} \bar{g}_{i}\left|h_{j i}\right|+D_{j} \bar{J}_{j}+\bar{d}_{j} \sum_{i=1}^{n} k_{i}\left|h_{j i}\right|<0,
\end{gathered}
$$

for $i=1,2 \ldots, n, j=1,2 \ldots, m$.

Proof. Let

$$
\begin{gathered}
\bar{u}(t)=\left(\bar{u}_{1}(t), \bar{u}_{2}(t), \ldots, \bar{u}_{n}(t)\right)^{T}, \\
\bar{v}(t)=\left(\bar{v}_{1}(t), \bar{v}_{2}(t), \ldots, \bar{v}_{m}(t)\right)^{T}
\end{gathered}
$$

be solution of system (3) with initial value (IV1), and let

$$
\begin{gathered}
u(t)=\left(u_{1}(t), u_{2}(t), \ldots, u_{n}(t)\right)^{T} \\
v(t)=\left(v_{1}(t), v_{2}(t), \ldots, v_{m}(t)\right)^{T}
\end{gathered}
$$

be solution of system (3) with any initial value (IV2).

From (33), we can obtain

$$
\begin{aligned}
\frac{d\left|z_{i}(t)\right|}{d t} & =\operatorname{sgn}\left(z_{i}(t)\right)\left(-z_{i}(t)+w_{i}(t)\right) \\
& \leq-\left|z_{i}(t)\right|+\left|w_{i}(t)\right|, \quad i=1,2 \ldots, n,
\end{aligned}
$$

$$
\begin{aligned}
\frac{d\left|w_{i}(t)\right|}{d t} & \\
=\operatorname{sgn}\left(w_{i}(t)\right)\{ & -\left(1-\alpha_{i}\right) z_{i}(t)-\left(\alpha_{i}-1\right) w_{i}(t) \\
& +a_{i}\left(u_{i}(t)\right) \\
& \times\left[\sum _ { j = 1 } ^ { m } c _ { i j } \left[f_{j}\left(v_{j}\left(t-\tau_{i j}(t)\right)\right)\right.\right. \\
& \left.-f_{j}\left(\bar{v}_{j}\left(t-\tau_{i j}(t)\right)\right)\right]
\end{aligned}
$$




$$
\begin{aligned}
& +\left(a_{i}\left(u_{i}(t)\right)-a_{i}\left(\bar{u}_{i}(t)\right)\right) \\
& \times\left[\sum_{j=1}^{m} c_{i j} f_{j}\left(\bar{v}_{j}\left(t-\tau_{i j}(t)\right)\right)+I_{i}(t)\right] \\
& -\left[a_{i}\left(u_{i}(t)\right) b_{i}\left(u_{i}(t)\right)\right. \\
& \left.\left.-a_{i}\left(\bar{u}_{i}(t)\right) b_{i}\left(\bar{u}_{i}(t)\right)\right]\right\} \\
& =\operatorname{sgn}\left(w_{i}(t)\right)\left\{-\left(1-\alpha_{i}\right) z_{i}(t)-\left(\alpha_{i}-1\right) w_{i}(t)\right. \\
& +a_{i}\left(u_{i}(t)\right) \\
& \times\left[\sum _ { j = 1 } ^ { m } c _ { i j } \left[f_{j}\left(v_{j}\left(t-\tau_{i j}(t)\right)\right)\right.\right. \\
& \left.\left.-f_{j}\left(\bar{v}_{j}\left(t-\tau_{i j}(t)\right)\right)\right]\right] \\
& +a_{i}^{\prime}\left(\xi_{i}\right) z_{i}(t) \\
& \times\left[\sum_{j=1}^{m} c_{i j} f_{j}\left(\bar{v}_{j}\left(t-\tau_{i j}(t)\right)\right)+I_{i}(t)\right] \\
& \left.-B_{i}^{\prime}\left(\bar{\xi}_{i}\right) z_{i}(t)\right\} \\
& \leq\left(\alpha_{i}-1-B_{i}^{\prime}\left(\bar{\xi}_{i}\right)\right)\left|z_{i}(t)\right|-\left(\alpha_{i}-1\right)\left|w_{i}(t)\right| \\
& +\bar{a}_{i} \sum_{j=1}^{m}\left|c_{i j}\right| l_{j}\left|p_{j}\left(t-\tau_{i j}(t)\right)\right| \\
& +A_{i}\left[\sum_{j=1}^{m}\left|c_{i j}\right| \bar{f}_{j}+\bar{I}_{i}\right]\left|z_{i}(t)\right| \\
& \leq\left[\alpha_{i}-1-T_{i}+A_{i}\left(\sum_{j=1}^{m}\left|c_{i j}\right| \bar{f}_{j}+\bar{I}_{i}\right)\right]\left|z_{i}(t)\right| \\
& -\left(\alpha_{i}-1\right)\left|w_{i}(t)\right|+\bar{a}_{i} \sum_{j=1}^{m}\left|c_{i j}\right| l_{j}\left|p_{j}\left(t-\tau_{i j}(t)\right)\right|,
\end{aligned}
$$

(60)

for $i=1,2, \ldots, n$.

From (34), we can obtain

$$
\begin{aligned}
\frac{d\left|p_{j}(t)\right|}{d t} & =\operatorname{sgn}\left(p_{j}(t)\right)\left(-p_{j}(t)+q_{j}(t)\right) \\
& \leq-\left|p_{j}(t)\right|+\left|q_{j}(t)\right|, \quad j=1,2, \ldots, m,
\end{aligned}
$$

$\frac{d\left|q_{j}(t)\right|}{d t}$$$
=\operatorname{sgn}\left(q_{j}(t)\right)\left\{-\left(1-\beta_{j}\right) p_{j}(t)-\left(\beta_{j}-1\right) q_{j}(t)\right.
$$$$
+d_{j}\left(v_{j}(t)\right)\left[\sum _ { i = 1 } ^ { n } h _ { j i } \left[g_{i}\left(u_{i}\left(t-\sigma_{j i}(t)\right)\right)\right.\right.
$$$$
\left.\left.-g_{i}\left(\bar{u}_{j}\left(t-\sigma_{j i}(t)\right)\right)\right]\right]
$$$$
+\left(d_{j}\left(v_{j}(t)\right)-d_{j}\left(\bar{v}_{j}(t)\right)\right)
$$$$
\cdot\left[\sum_{i=1}^{n} h_{j i} g_{i}\left(\bar{u}_{i}\left(t-\sigma_{j i}(t)\right)\right)+J_{j}(t)\right]
$$$$
-\left[d_{j}\left(v_{j}(t)\right) e_{j}\left(v_{j}(t)\right)\right.
$$$$
\left.\left.-d_{j}\left(\bar{v}_{j}(t)\right) e_{j}\left(\bar{v}_{j}(t)\right)\right]\right\}
$$$$
=\operatorname{sgn}\left(q_{j}(t)\right)\left\{-\left(1-\beta_{j}\right) p_{j}(t)-\left(\beta_{j}-1\right) q_{j}(t)\right.
$$$$
+d_{j}\left(v_{j}(t)\right)\left[\sum _ { i = 1 } ^ { n } h _ { j i } \left[g_{i}\left(u_{i}\left(t-\sigma_{j i}(t)\right)\right)\right.\right.
$$$$
\left.\left.-g_{i}\left(\bar{u}_{j}\left(t-\sigma_{j i}(t)\right)\right)\right]\right]
$$$$
+d_{j}^{\prime}\left(\xi_{j}^{*}\right) p_{j}(t)\left[\sum_{i=1}^{n} h_{j i} g_{i}\left(\bar{u}_{i}\left(t-\sigma_{j i}(t)\right)\right)\right.
$$$$
\left.+J_{j}(t)\right]
$$$$
\left.-E_{j}^{\prime}\left(\bar{\xi}_{j}^{*}\right) p_{j}(t)\right\}
$$$$
\leq\left(\beta_{j}-1-E_{j}^{\prime}\left(\bar{\xi}_{j}^{*}\right)\right)\left|p_{j}(t)\right|-\left(\beta_{j}-1\right)\left|q_{j}(t)\right|
$$$$
+\bar{d}_{j} \sum_{i=1}^{n}\left|h_{j i}\right| k_{i}\left|z_{i}\left(t-\sigma_{j i}(t)\right)\right|+D_{j}\left|p_{j}(t)\right|
$$$$
\times\left(\sum_{i=1}^{n}\left|h_{j i}\right| \bar{g}_{i}+\bar{J}_{j}\right)
$$

$$
\begin{aligned}
& \leq\left[\beta_{j}-1-T_{j}^{*}+D_{j}\left(\sum_{i=1}^{n}\left|h_{j i}\right| \bar{g}_{i}+\bar{J}_{j}\right)\right]\left|p_{j}(t)\right| \\
& \quad-\left(\beta_{j}-1\right)\left|q_{j}(t)\right|+\bar{d}_{j} \sum_{i=1}^{n}\left|h_{j i}\right| k_{i}\left|z_{i}\left(t-\sigma_{j i}(t)\right)\right|,
\end{aligned}
$$

for $j=1,2, \ldots, m$. 
From (60) and (62), we can obtain

$$
\begin{aligned}
\left|w_{i}(t)\right| \leq & e^{\left(1-\alpha_{i}\right) t}\left|w_{i}(0)\right| \\
& +\left[\alpha_{i}-1-T_{i}+A_{i}\left(\sum_{j=1}^{m}\left|c_{i j}\right| \bar{f}_{j}+\bar{I}_{i}\right)\right] \\
& \times \int_{0}^{t} e^{\left(\alpha_{i}-1\right)(s-t)}\left|z_{i}(s)\right| d s \\
& +\bar{a}_{i} \sum_{j=1}^{m}\left|c_{i j}\right| l_{j} \int_{0}^{t} e^{\left(\alpha_{i}-1\right)(s-t)}\left|p_{j}\left(s-\tau_{i j}(t)\right)\right| d s
\end{aligned}
$$

for $i=1,2, \ldots, n$.

$$
\begin{aligned}
\left|q_{j}(t)\right| \leq & e^{\left(1-\beta_{j}\right) t}\left|q_{j}(0)\right| \\
& +\left[\beta_{j}-1-T_{j}^{*}+D_{j}\left(\sum_{i=1}^{n}\left|h_{j i}\right| \bar{g}_{i}+\bar{J}_{j}\right)\right] \\
& \times \int_{0}^{t} e^{\left(\beta_{j}-1\right)(s-t)}\left|p_{j}(s)\right| d s \\
& +\bar{d}_{j} \sum_{i=1}^{n}\left|h_{j i}\right| k_{i} \int_{0}^{t} e^{\left(\beta_{j}-1\right)(s-t)}\left|z_{i}\left(s-\sigma_{j i}(t)\right)\right| d s
\end{aligned}
$$

for $j=1,2, \ldots, m$.

We consider the functions $g_{i}(\xi), h_{j}(\eta)$ given by

$$
\begin{gathered}
g_{i}(\xi)=\xi-T_{i}+A_{i} \sum_{j=1}^{m} \bar{f}_{j}\left|c_{i j}\right|+A_{i} \bar{I}_{i}+\bar{a}_{i} \sum_{j=1}^{m} l_{j}\left|c_{i j}\right| e^{\xi \tau}, \\
h_{j}(\eta)=\eta-T_{j}^{*}+D_{j} \sum_{i=1}^{n} \bar{g}_{i}\left|h_{j i}\right|+D_{j} \bar{J}_{j}+\bar{d}_{j} \sum_{i=1}^{n} k_{i}\left|h_{j i}\right| e^{\eta \sigma},
\end{gathered}
$$

for $i=1,2, \ldots, n, j=1,2, \ldots, m$.

Obviously

$$
\begin{array}{cll}
\frac{d g_{i}(\xi)}{d \xi}>0, & \lim _{\xi \rightarrow+\infty} g_{i}(\xi)=+\infty, & g_{i}(0)<0, \\
\frac{d h_{j}(\eta)}{d \eta}>0, & \lim _{\eta \rightarrow+\infty} h_{j}(\eta)=+\infty, & h_{j}(0)<0,
\end{array}
$$

for $i=1,2, \ldots, n, j=1,2, \ldots, m$.

Therefore, there exist constants $\xi_{i}, \eta_{j} \in(0,+\infty)$, such that

$$
\begin{gathered}
g_{i}\left(\xi_{i}\right)=0, \quad i=1,2, \ldots, n, \\
h_{j}\left(\eta_{j}\right)=0, \quad j=1,2, \ldots, m .
\end{gathered}
$$

We choose $\bar{\xi}=\min \left\{\xi_{1}, \xi_{2}, \ldots, \xi_{n}, \eta_{1}, \eta_{2}, \ldots, \eta_{m}\right\}$, then $\bar{\xi}>0$; when $0<\delta<\bar{\xi}$, we have

$$
\begin{gathered}
\delta-T_{i}+A_{i} \sum_{j=1}^{m} \bar{f}_{j}\left|c_{i j}\right|+A_{i} \bar{I}_{i}+\bar{a}_{i} \sum_{j=1}^{m} l_{j}\left|c_{i j}\right| e^{\delta \tau}<0, \\
\delta-T_{j}^{*}+D_{j} \sum_{i=1}^{n} \bar{g}_{i}\left|h_{j i}\right|+D_{j} \bar{J}_{j}+\bar{d}_{j} \sum_{i=1}^{n} k_{i}\left|h_{j i}\right| e^{\delta \sigma}<0,
\end{gathered}
$$

for $i=1,2, \ldots, n, j=1,2, \ldots, m$.

Since the initial values $\varphi_{u i}(s), \psi_{u i}(s), \varphi_{v j}(s)$, and $\psi_{v j}(s)$ are bounded and continuous functions, then there exist $N_{1}, N_{2}$, $N_{3}$, and $N_{4}>0$, such that $\left|\varphi_{u i}(t)\right| \leq N_{1},\left|\psi_{u i}(t)\right| \leq N_{2}$, $\left|\varphi_{v j}(t)\right| \leq N_{3},\left|\psi_{v j}(4)\right| \leq N_{4}, i=1,2, \ldots, n, j=1,2, \ldots, m$, and $t \in[-\gamma, 0], \gamma=\max \{\sigma, \tau\}$.

Let $L=\max \left\{N_{1}+N_{2}, N_{3}+N_{4}\right\}$; we will show that, for any sufficiently small constant $\varepsilon>0$ and $0<\delta<\bar{\xi}$,

$$
\begin{array}{ll}
\left|z_{i}(t)\right|<(L+\varepsilon) e^{-\delta t}, & \left|w_{i}(t)\right|<(L+\varepsilon) e^{-\delta t}, \\
\left|p_{j}(t)\right|<(L+\varepsilon) e^{-\delta t}, & \left|q_{j}(t)\right|<(L+\varepsilon) e^{-\delta t},
\end{array}
$$

for $i=1,2, \ldots, n, j=1,2, \ldots, m$, and $t \geq 0$.

Considering proof of contradiction, if (69) does not hold, there are 15 possible situations; here we only discuss the following four cases; that is,

$$
\begin{gathered}
\left|z_{k}\left(t_{1}\right)\right|=(L+\varepsilon) e^{-\delta t_{1}}, \quad \frac{d^{+}\left|z_{k}\left(t_{1}\right)\right|}{d t} \geq 0, \\
\left|z_{i}(t)\right|<(L+\varepsilon) e^{-\delta t}, \quad t \in\left[0, t_{1}\right), \\
\left|w_{i}(t)\right|<(L+\varepsilon) e^{-\delta t}, \quad t \in\left[0, t_{1}\right], \\
\left|p_{j}(t)\right|<(L+\varepsilon) e^{-\delta t}, \quad t \in\left[0, t_{1}\right], \\
\left|q_{j}(t)\right|<(L+\varepsilon) e^{-\delta t}, \quad t \in\left[0, t_{1}\right], \\
\left|w_{k}\left(t_{1}\right)\right|=(L+\varepsilon) e^{-\delta t_{1}}, \\
\left|z_{i}(t)\right|<(L+\varepsilon) e^{-\delta t}, \quad t \in\left[0, t_{1}\right], \\
\left|w_{i}(t)\right|<(L+\varepsilon) e^{-\delta t}, \quad t \in\left[0, t_{1}\right), \\
\left|p_{j}(t)\right|<(L+\varepsilon) e^{-\delta t}, \quad t \in\left[0, t_{1}\right], \\
\left|q_{j}(t)\right|<(L+\varepsilon) e^{-\delta t}, \quad t \in\left[0, t_{1}\right],
\end{gathered}
$$

$$
\begin{array}{cl}
\left|p_{l}\left(t_{1}\right)\right|=(L+\varepsilon) e^{-\delta t_{1}}, & \frac{d^{+}\left|p_{l}\left(t_{1}\right)\right|}{d t} \geq 0, \\
\left|z_{i}(t)\right|<(L+\varepsilon) e^{-\delta t}, \quad t \in\left[0, t_{1}\right], \\
\left|w_{i}(t)\right|<(L+\varepsilon) e^{-\delta t}, \quad t \in\left[0, t_{1}\right], \\
\left|p_{j}(t)\right|<(L+\varepsilon) e^{-\delta t}, \quad t \in\left[0, t_{1}\right), \\
\left|q_{j}(t)\right|<(L+\varepsilon) e^{-\delta t}, \quad t \in\left[0, t_{1}\right],
\end{array}
$$


or

$$
\begin{gathered}
\left|q_{i}\left(t_{1}\right)\right|=(L+\varepsilon) e^{-\delta t_{1}}, \\
\left|z_{i}(t)\right|<(L+\varepsilon) e^{-\delta t}, \quad t \in\left[0, t_{1}\right], \\
\left|w_{i}(t)\right|<(L+\varepsilon) e^{-\delta t}, \quad t \in\left[0, t_{1}\right], \\
\left|p_{j}(t)\right|<(L+\varepsilon) e^{-\delta t}, \quad t \in\left[0, t_{1}\right], \\
\left|q_{j}(t)\right|<(L+\varepsilon) e^{-\delta t}, \quad t \in\left[0, t_{1}\right),
\end{gathered}
$$

where $k \in\{1,2, \ldots, n\}, l \in\{1,2, \ldots, m\}$, and $t_{1} \geq 0, i=$ $1,2, \ldots, n$, and $j=1,2, \ldots, m$.

Therefore, by (59) and (70), we have

$$
\begin{aligned}
\frac{d^{+}\left|z_{k}\left(t_{1}\right)\right|}{d t} & \leq-\left|z_{k}\left(t_{1}\right)\right|+\left|w_{k}\left(t_{1}\right)\right| \\
& <-(L+\varepsilon) e^{-\delta t_{1}}+(L+\varepsilon) e^{-\delta t_{1}}=0,
\end{aligned}
$$

which is a contradiction with $d^{+}\left|z_{k}\left(t_{1}\right)\right| / d t \geq 0$.

By (63) and (71), we obtain

$$
\begin{aligned}
& \left|w_{k}\left(t_{1}\right)\right|=(L+\varepsilon) e^{-\delta t_{1}} \\
& \leq e^{\left(1-\alpha_{k}\right) t_{1}}\left|w_{k}(0)\right|+\left[\alpha_{k}-1-T_{k}+A_{k} \sum_{j=1}^{m} \bar{f}_{j}\left|c_{k j}\right|+A_{k} \bar{I}_{k}\right] \\
& \times \int_{0}^{t_{1}} e^{\left(\alpha_{k}-1\right)\left(s-t_{1}\right)}\left|z_{k}(s)\right| d s+\bar{a}_{k} \sum_{j=1}^{m} l_{j}\left|c_{k j}\right| \\
& \times \int_{0}^{t_{1}} e^{\left(\alpha_{k}-1\right)\left(s-t_{1}\right)}\left|p_{j}\left(s-\tau_{k j}(s)\right)\right| d s \\
& \leq(L+\varepsilon)\left\{e^{\left(1-\alpha_{k}\right) t_{1}}+\left[\alpha_{k}-1-T_{k}+A_{k} \sum_{j=1}^{m} \bar{f}_{j}\left|c_{k j}\right|+A_{k} \bar{I}_{k}\right]\right. \\
& \times \int_{0}^{t_{1}} e^{\left(\alpha_{k}-1\right)\left(s-t_{1}\right)-\delta s} d s+\bar{a}_{k} \sum_{j=1}^{m} l_{j}\left|c_{k j}\right| \\
& \left.\times \int_{0}^{t_{1}} e^{\left(\alpha_{k}-1\right)\left(s-t_{1}\right)-\delta s+\delta \tau_{k j}(s)} d s\right\} \\
& \quad\left\{\begin{array}{c}
\left.+A_{k} \bar{I}_{k}+\bar{a}_{k} \sum_{j=1}^{m} l_{j}\left|c_{k j}\right| e^{\tau \delta}\right] \\
\leq(L+\varepsilon) \\
\left\{e^{\left(1-\alpha_{k}\right) t_{1}}+\left[\alpha_{k}-1-T_{k}+A_{k} \sum_{j=1}^{m} \bar{f}_{j}\left|c_{k j}\right|\right.\right.
\end{array}\right. \\
& \qquad \begin{array}{c}
\alpha_{k}-1-\delta \\
\left(1-\alpha_{k}\right) t_{1}
\end{array} .
\end{aligned}
$$

Since

$$
\begin{array}{r}
\alpha_{k}-1-\delta> \\
\alpha_{k}-1-T_{k}+A_{k} \sum_{j=1}^{m} \bar{f}_{j}\left|c_{k j}\right| \\
+A_{k} \bar{I}_{k}+\bar{a}_{k} \sum_{j=1}^{m} l_{j}\left|c_{k j}\right| e^{\tau \delta}>0,
\end{array}
$$

we have

$$
\frac{\left[\alpha_{k}-1-T_{k}+A_{k} \sum_{j=1}^{m} \bar{f}_{j}\left|c_{k j}\right|+A_{k} \bar{I}_{k}+\bar{a}_{k} \sum_{j=1}^{m} l_{j}\left|c_{k j}\right| e^{\tau \delta}\right]}{\left(\alpha_{k}-1-\delta\right)}
$$

$<1$.

From (75), we have

$$
L+\varepsilon<L+\varepsilon,
$$

which is a contradiction.

Therefore, by (61) and (75), we have

$$
\begin{aligned}
\frac{d^{+}\left|p_{l}\left(t_{1}\right)\right|}{d t} & \leq-\left|p_{l}\left(t_{1}\right)\right|+\left|q_{l}\left(t_{1}\right)\right| \\
& <-(L+\varepsilon) e^{-\delta t_{1}}+(L+\varepsilon) e^{-\delta t_{1}}=0,
\end{aligned}
$$

which is a contradiction with $d^{+}\left|p_{l}\left(t_{1}\right)\right| / d t \geq 0$.

By (70) and (80), we obtain

$$
\begin{aligned}
& \left|q_{l}\left(t_{1}\right)\right| \\
& =(L+\varepsilon) e^{-\delta t_{1}} \leq e^{\left(1-\beta_{l}\right) t_{1}}\left|q_{l}(0)\right| \\
& +\left[\beta_{l}-1-T_{l}^{*}+D_{l} \sum_{i=1}^{n} \bar{g}_{i}\left|h_{l i}\right|+D_{l} \bar{J}_{l}\right] \\
& \quad \times \int_{0}^{t_{1}} e^{\left(\beta_{l}-1\right)\left(s-t_{1}\right)}\left|p_{l}(s)\right| d s+\bar{d}_{l} \sum_{i=1}^{n} k_{i}\left|h_{l i}\right| \\
& \quad \times \int_{0}^{t_{1}} e^{\left(\beta_{l}-1\right)\left(s-t_{1}\right)}\left|z_{i}\left(s-\sigma_{l i}(s)\right)\right| d s \\
& \leq(L+\varepsilon)\left\{e^{\left(1-\beta_{l}\right) t_{1}}+\left[\beta_{l}-1-T_{l}^{*}+D_{l} \sum_{i=1}^{n} \bar{g}_{i}\left|h_{l i}\right|+D_{l} \bar{J}_{l}\right]\right. \\
& \quad \times \int_{0}^{t_{1}} e^{\left(\beta_{l}-1\right)\left(s-t_{1}\right)-\delta s} d s+\bar{d}_{l} \sum_{i=1}^{n} k_{i}\left|h_{l i}\right| \\
& \left.\quad \times \int_{0}^{t_{1}} e^{\left(\beta_{l}-1\right)\left(s-t_{1}\right)-\delta s+\delta \sigma_{l i}(s)} d s\right\}
\end{aligned}
$$




$$
\begin{gathered}
\leq(L+\varepsilon)\left\{e^{\left(1-\beta_{l}\right) t_{1}}+\left[\beta_{l}-1-T_{l}^{*}+D_{l} \sum_{i=1}^{n} \bar{g}_{i}\left|h_{l i}\right|\right.\right. \\
\left.+D_{l} \bar{J}_{l}+\bar{d}_{l} \sum_{i=1}^{n} k_{i}\left|h_{l i}\right| e^{\sigma \delta}\right] \\
\left.\times \frac{e^{-\delta t_{1}}-e^{\left(1-\beta_{l}\right) t_{1}}}{\beta_{l}-1-\delta}\right\} .
\end{gathered}
$$

Since

$$
\begin{aligned}
\beta_{l}-1-\delta> & \beta_{l}-1-T_{l}^{*} \\
& +D_{l} \sum_{i=1}^{n} \bar{g}_{i}\left|h_{l i}\right|+D_{l} \bar{J}_{l}+\bar{d}_{l} \sum_{i=1}^{n} k_{i}\left|h_{l i}\right| e^{\sigma \delta}>0,
\end{aligned}
$$

we have

$$
\frac{\left[\beta_{l}-1-T_{l}^{*}+D_{l} \sum_{i=1}^{n} \bar{g}_{i}\left|h_{l i}\right|+D_{l} \bar{J}_{l}+\bar{d}_{l} \sum_{i=1}^{n} k_{i}\left|h_{l i}\right| e^{\sigma \delta}\right]}{\left(\beta_{l}-1-\delta\right)}
$$

$<1$.

From (80), we have

$$
L+\varepsilon<L+\varepsilon,
$$

which is a contradiction.

Similarly, we can prove that the other 11 possible situations; thus (69) holds; let $\varepsilon \rightarrow 0$; we have

$$
\begin{array}{ll}
\left|z_{i}(t)\right| \leq L e^{-\delta t}, & \left|w_{i}(t)\right| \leq L e^{-\delta t}, \\
\left|p_{j}(t)\right| \leq L e^{-\delta t}, & \left|q_{j}(t)\right| \leq L e^{-\delta t}
\end{array}
$$

for $i=1,2, \ldots, n, j=1,2, \ldots, m$, and $t \geq 0$.

From (84), there exist constants $\delta>0$ and $M>0$ such that

$$
\begin{aligned}
& \sum_{i=1}^{n}\left(u_{i}(t)-\bar{u}_{i}(t)\right)^{2}+\sum_{j=1}^{m}\left(v_{j}(t)-\bar{v}_{j}(t)\right)^{2} \\
& <M e^{-\delta t}\left[\left\|\varphi_{u i}-\bar{\varphi}_{u i}\right\|^{2}+\left\|\varphi_{v j}-\bar{\varphi}_{v j}\right\|^{2}\right], \quad t>0 .
\end{aligned}
$$

Using (85), similarly with the proof of Theorem 3, we know that system (3) has one $\omega$-periodic solution, which is exponentially stable.

\section{Numerical Examples}

In this section, we give two examples for showing our results.
Example 1. We consider the following inertial CohenGrossberg-type BAM neural networks with time delays $(n=$ $m=3)$ :

$$
\begin{aligned}
& \frac{d^{2} u_{i}(t)}{d t^{2}} \\
& =-\alpha_{i} \frac{d u_{i}(t)}{d t}-a_{i}\left(u_{i}(t)\right) \\
& \times\left[b_{i}\left(u_{i}(t)\right)-\sum_{j=1}^{3} c_{i j} f_{j}\left(v_{j}\left(t-\tau_{i j}(t)\right)\right)-I_{i}(t)\right], \\
& \frac{d^{2} v_{j}(t)}{d t^{2}} \\
& =-\beta_{j} \frac{d v_{j}(t)}{d t}-d_{j}\left(v_{j}(t)\right) \\
& \times\left[e_{j}\left(v_{j}(t)\right)-\sum_{i=1}^{3} h_{j i} g_{i}\left(u_{i}\left(t-\sigma_{j i}(t)\right)\right)-J_{j}(t)\right],
\end{aligned}
$$

for $i, j=1,2,3$, where

$$
\begin{aligned}
& \alpha_{1}=1.7, \quad \alpha_{2}=1.9, \quad \alpha_{3}=1.8, \quad \beta_{1}=2.3, \\
& \beta_{2}=2, \quad \beta_{3}=2.2, \quad c_{11}=\frac{1}{16}, \quad c_{12}=\frac{1}{16}, \\
& c_{13}=\frac{1}{24}, \quad c_{21}=\frac{1}{8}, \quad c_{22}=\frac{1}{8}, \quad c_{23}=\frac{1}{32}, \\
& c_{31}=\frac{1}{32}, \quad c_{32}=\frac{1}{32}, \quad c_{33}=\frac{1}{64}, \quad h_{11}=\frac{1}{32}, \\
& h_{12}=\frac{1}{32}, \quad h_{13}=\frac{1}{64}, \quad h_{21}=\frac{1}{32}, \quad h_{22}=\frac{1}{32} \text {, } \\
& h_{23}=\frac{1}{32}, \quad h_{31}=\frac{1}{64}, \quad h_{32}=\frac{1}{64}, \quad h_{33}=\frac{1}{32} \text {, } \\
& a_{1}\left(u_{1}\right)=2+\frac{1}{1+u_{1}^{2}}, \quad a_{2}\left(u_{2}\right)=2-\frac{1}{1+u_{2}^{2}}, \\
& a_{3}\left(u_{3}\right)=1.6+\frac{2}{1+u_{3}^{2}} \\
& b_{1}\left(u_{1}\right)=\frac{8}{15} u_{1}, \quad b_{2}\left(u_{2}\right)=\frac{1}{2} u_{2}, \quad b_{3}\left(u_{3}\right)=\frac{10}{20} u_{3} \text {, } \\
& d_{1}\left(v_{1}\right)=1+\frac{1}{1+v_{1}^{2}}, \quad d_{2}\left(v_{2}\right)=2-\frac{1}{1+v_{2}^{2}}, \\
& d_{3}\left(v_{3}\right)=2+\frac{1}{1+v_{3}^{2}}, \\
& e_{1}\left(v_{1}\right)=\frac{4}{7} v_{1}, \quad e_{2}\left(v_{2}\right)=\frac{1}{3} v_{2}, \quad e_{3}\left(v_{3}\right)=\frac{4}{15} v_{3}, \\
& f_{j}\left(v_{j}\right)=\frac{e^{v_{j}}-e^{-v_{j}}}{8\left(e^{v_{j}}+e^{-v_{j}}\right)}, \quad g_{i}\left(u_{i}\right)=\frac{e^{u_{i}}}{8\left(e^{u_{i}}+e^{-u_{i}}\right)},
\end{aligned}
$$




$$
\begin{gathered}
\tau_{i j}=\frac{1+\sin t}{6}, \quad \sigma_{j i}=\frac{1+\cos t}{6}, \\
I_{i}(t)=\frac{1}{32}(2+\sin t), \quad J_{j}(t)=\frac{1}{32}(2+\cos t), \\
i, j=1,2,3 .
\end{gathered}
$$

Obviously,

$$
\begin{aligned}
& 2 \leq a_{1}\left(u_{1}\right) \leq 3, \quad\left|a_{1}^{\prime}\left(u_{1}\right)\right| \leq 1, \quad 1 \leq a_{2}\left(u_{2}\right) \leq 2, \\
& \left|a_{2}^{\prime}\left(u_{2}\right)\right| \leq 1, \quad 1.6 \leq a_{3}\left(u_{3}\right) \leq 3.6, \quad\left|a_{3}^{\prime}\left(u_{3}\right)\right| \leq 2 \text {, } \\
& 1 \leq d_{1}\left(v_{1}\right) \leq 2, \quad\left|d_{1}^{\prime}\left(v_{1}\right)\right| \leq 1, \quad 1 \leq d_{2}\left(v_{2}\right) \leq 2, \\
& \left|d_{2}^{\prime}\left(v_{2}\right)\right| \leq 1, \quad 2 \leq d_{3}\left(v_{3}\right) \leq 3, \quad\left|d_{3}^{\prime}\left(v_{3}\right)\right| \leq 1, \\
& b_{1}^{\prime}\left(u_{1}\right)=\frac{8}{15}, \quad b_{2}^{\prime}\left(u_{2}\right)=\frac{1}{2}, \quad b_{3}^{\prime}\left(u_{3}\right)=\frac{10}{20}, \\
& e_{1}^{\prime}\left(v_{1}\right)=\frac{4}{7}, \quad e_{2}^{\prime}\left(v_{2}\right)=\frac{1}{3}, \quad e_{3}^{\prime}\left(v_{3}\right)=\frac{4}{15}, \\
& \left|f_{j}(x)-f_{j}(y)\right| \leq \frac{|x-y|}{8}, \quad\left|f_{j}(x)\right| \leq \frac{1}{8}, \\
& j=1,2,3, \quad x, y \in R, \\
& \left|g_{i}(x)-g_{i}(y)\right| \leq \frac{|x-y|}{16}, \quad\left|g_{i}(x)\right| \leq \frac{1}{8}, \\
& i=1,2,3, \quad x, y \in R, \\
& 0 \leq \tau_{i j} \leq \frac{1}{3}, \quad 0 \leq \sigma_{j i}=\frac{1}{3}, \quad i=j=1,2,3, \\
& \tau_{i j}^{\prime} \leq \frac{1}{6}, \quad \sigma_{j i}^{\prime}=\frac{1}{6}, \quad i=j=1,2,3, \\
& \frac{1}{32}<I_{i}(t)<\frac{3}{32}, \quad \frac{1}{32}<J_{j}(t)<\frac{3}{32}, \quad i, j=1,2,3, \\
& B_{1}\left(u_{1}\right)=a_{1}\left(u_{1}\right) b_{1}\left(u_{1}\right)=\frac{8}{15}\left(2 u_{1}+\frac{u_{1}}{1+u_{1}^{2}}\right), \\
& 1 \leq B_{1}^{\prime}\left(u_{1}\right)=\frac{8}{15}\left(2+\frac{1-u_{1}^{2}}{\left(1+u_{1}^{2}\right)^{2}}\right) \leq \frac{8}{5}, \\
& B_{2}\left(u_{2}\right)=a_{2}\left(u_{2}\right) b_{2}\left(u_{2}\right)=\frac{1}{2}\left(2 u_{2}-\frac{u_{2}}{1+u_{2}^{2}}\right) \text {, } \\
& \frac{1}{2} \leq B_{2}^{\prime}\left(u_{2}\right)=\frac{1}{2}\left(2+\frac{u_{2}^{2}-1}{\left(1+u_{2}^{2}\right)^{2}}\right) \leq \frac{17}{16} \\
& B_{3}\left(u_{3}\right)=a_{3}\left(u_{3}\right) b_{3}\left(u_{3}\right)=\frac{10}{27}\left(1.6 u_{3}+\frac{2 u_{3}}{1+u_{3}^{2}}\right) \text {, } \\
& \frac{1}{2} \leq B_{3}^{\prime}\left(u_{3}\right)=\frac{10}{27}\left(1.6+2 \frac{1-u_{3}^{2}}{\left(1+u_{3}^{2}\right)^{2}}\right) \leq \frac{4}{3},
\end{aligned}
$$

$$
\begin{gathered}
E_{1}\left(v_{1}\right)=d_{1}\left(v_{1}\right) e_{1}\left(v_{1}\right)=\frac{4}{7}\left(v_{1}+\frac{v_{1}}{1+v_{1}^{2}}\right), \\
\frac{1}{2} \leq E_{1}^{\prime}\left(v_{1}\right)=\frac{4}{7}\left(1+\frac{1-v_{1}^{2}}{\left(1+v_{1}^{2}\right)^{2}}\right) \leq \frac{8}{7}, \\
E_{2}\left(v_{2}\right)=d_{2}\left(v_{2}\right) e_{2}\left(v_{2}\right)=\frac{1}{3}\left(2 v_{2}-\frac{v_{2}}{1+v_{2}^{2}}\right), \\
\frac{1}{3} \leq E_{2}^{\prime}\left(v_{2}\right)=\frac{1}{3}\left(2+\frac{v_{2}^{2}-1}{\left(1+v_{2}^{2}\right)^{2}}\right) \leq \frac{17}{24}, \\
E_{3}\left(v_{3}\right)=d_{3}\left(v_{3}\right) e_{3}\left(v_{3}\right)=\frac{4}{15}\left(2 v_{3}+\frac{v_{3}}{1+v_{3}^{2}}\right), \\
\frac{1}{2} \leq E_{3}^{\prime}\left(v_{3}\right)=\frac{4}{15}\left(2+\frac{1-v_{3}^{2}}{\left(1+v_{3}^{2}\right)^{2}}\right) \leq \frac{4}{5} .
\end{gathered}
$$

By assumptions $\left(H_{1}\right)-\left(H_{5}\right)$, we select

$$
\begin{aligned}
& \underline{a}_{1}=2, \quad \bar{a}_{1}=3, \quad \underline{a}_{2}=1, \quad \bar{a}_{2}=2, \quad \underline{a}_{3}=1.6, \\
& \bar{a}_{3}=3.6, \quad A_{1}=1, \quad A_{2}=1, \quad A_{3}=2, \\
& \underline{d}_{1}=1, \quad \bar{d}_{1}=2, \quad \underline{d}_{2}=1, \quad \bar{d}_{2}=2, \\
& \underline{d}_{3}=2, \quad \bar{d}_{3}=3, \quad D_{1}=1, \quad D_{2}=1, \quad D_{3}=1 \text {, } \\
& \underline{b}_{1}=\bar{b}_{1}=\frac{8}{15}, \quad \underline{b}_{2}=\bar{b}_{2}=\frac{1}{2}, \quad \underline{b}_{3}=\bar{b}_{3}=\frac{10}{27}, \\
& \underline{e}_{1}=\bar{e}_{1}=\frac{4}{7}, \quad \underline{e}_{2}=\bar{e}_{2}=\frac{1}{3}, \quad \underline{e}_{3}=\bar{e}_{3}=\frac{4}{15}, \\
& l_{j}=\bar{f}_{j}=\frac{1}{8}, \quad k_{i}=\frac{1}{16}, \quad \bar{g}_{i}=\frac{1}{8}, \quad I_{i}=\frac{1}{32}, \\
& \bar{I}_{i}=\frac{3}{32}, \quad J_{j}=\frac{1}{32}, \quad \bar{J}_{j}=\frac{3}{32}, \quad i, j=1,2,3, \\
& T_{1}=1, \quad k_{1}=\frac{8}{5}, \quad T_{2}=\frac{1}{2}, \quad k_{2}=\frac{17}{16}, \\
& T_{3}=\frac{1}{2}, \quad k_{3}=\frac{4}{3}, \quad T_{1}^{*}=\frac{1}{2}, \quad k_{1}^{*}=\frac{8}{7}, \\
& T_{2}^{*}=\frac{1}{3}, \quad k_{2}^{*}=\frac{17}{24}, \quad T_{3}^{*}=\frac{1}{2}, \quad k_{3}^{*}=\frac{4}{5}, \\
& \tau=\frac{1}{3}, \quad \sigma=\frac{1}{3}, \quad \bar{\tau}_{i j}=\frac{1}{6}, \quad \bar{\sigma}_{j i}=\frac{1}{3} .
\end{aligned}
$$

Thus, hypotheses $\left(H_{1}\right)-\left(H_{5}\right)$ hold.

For numerical simulation, the following ten any intial values are given:

$$
\begin{gathered}
{\left[\varphi_{u 1}(0), \varphi_{u 2}(0), \varphi_{u 3}(0), \psi_{u 1}(0), \psi_{u 2}(0), \psi_{u 3}(0),\right.} \\
\left.\varphi_{v 1}(0), \varphi_{v 2}(0), \varphi_{v 3}(0), \psi_{v 1}(0), \psi_{v 2}(0), \psi_{v 3}(0)\right]
\end{gathered}
$$




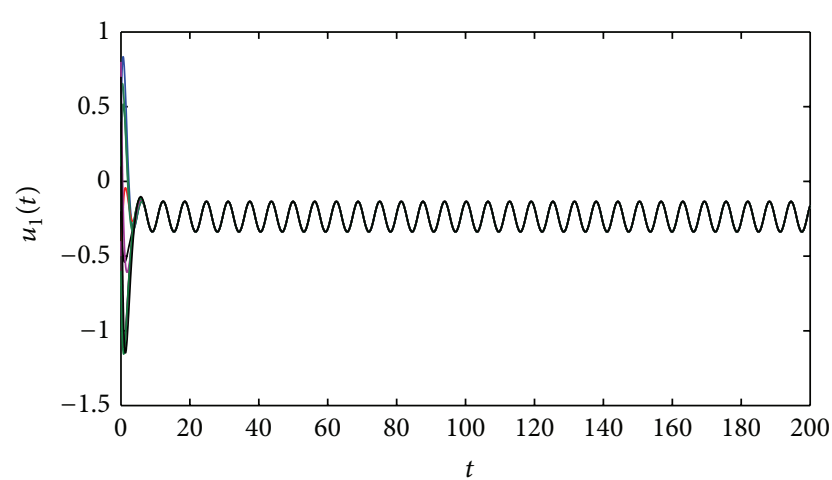

FIgURE 1: Transient response of state variables $u_{1}(t)$ of Example 1.

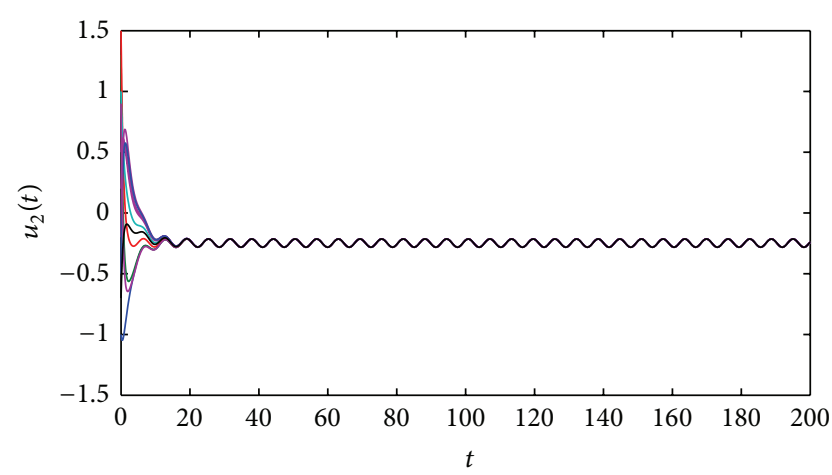

FIGURE 2: Transient response of state variables $u_{2}(t)$ of Example 1.

$=[0.4 ;-1 ; 1.5 ; 2 ;-1.3 ; 2 ; 0.5 ; 1.8 ; 3 ; 2 ;-3 ; 2]$

$[0.1 ; 1.3 ; 0.5 ; 1.5 ;-2 ; 1.5 ; 1 ; 1.3 ; 1 ; 1.5 ;-2 ; 1.5]$;

$[-0.2 ; 1.5 ;-1 ;-1 ;-1 ;-1 ;-1.5 ; 1.5 ;-1 ; 1.5 ;-2 ; 1.5]$;

$[-0.7 ; 1 ; 1.8 ; 0.5 ; 0 ;-1.3 ; 0.2 ;-1.3 ; 2 ; 1.5 ;-2 ; 1.5]$

$[-0.4 ; 0.5 ; 0.8 ;-2.3 ; 1 ;-2.3 ;-0.5 ;-1.5 ;-2 ; 1.5 ;-2 ; 1.5]$;

$[0.5 ;-0.2 ; 1 ; 1.3 ; 2 ; 1.3 ; 1.8 ; 2 ; 0.4 ; 1.5 ;-2 ; 1.5]$;

$[-0.5 ; 0.2 ; 2 ;-2.3 ; 1.3 ;-2.3 ; 0.6 ;-0.2 ;-0.4 ; 1.5 ;-2 ; 1.5]$

$[0.8 ;-0.5 ;-2 ;-1.3 ; 2 ;-1.3 ;-1.5 ; 0.5 ;-2.6 ; 1.5 ;-2 ; 1.5]$

$[-0.6 ; 0.9 ;-0.5 ;-2.3 ;-2 ;-2.3 ;-1.2$

$-0.5 ;-2.3 ; 1.5 ;-2 ; 1.5]$

$[0.7 ;-0.7 ;-1.5 ;-3 ; 0.5 ;-1 ;-1.1 ;-1.8 ;-0.2 ; 1.5 ;-2 ; 1.5]$.

Figures 1, 2, 3, 4, 5, and 6 depict the time responses of state variables of $u_{1}(t), u_{2}(t)$, and $u_{3}(t)$ and $v_{1}(t), v_{2}(t)$, and $v_{3}(t)$, of the system in Example 1, respectively.

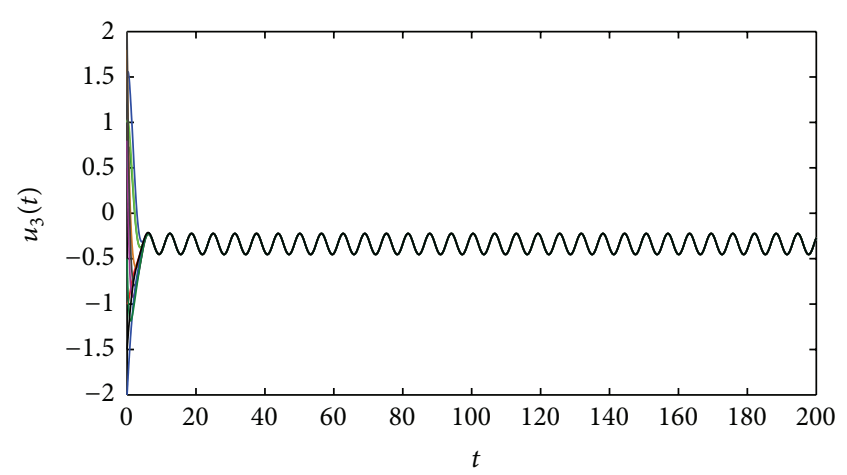

Figure 3: Transient response of state variables $u_{3}(t)$ of Example 1.

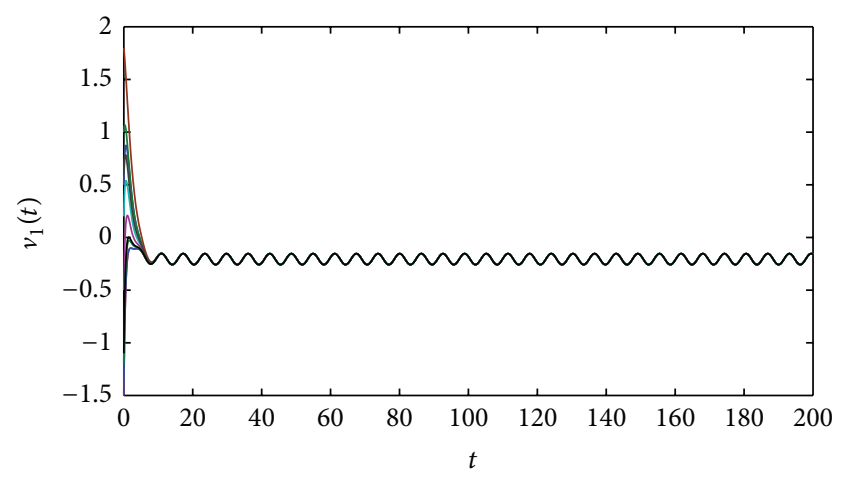

Figure 4: Transient response of state variables $v_{1}(t)$ of Example 1.

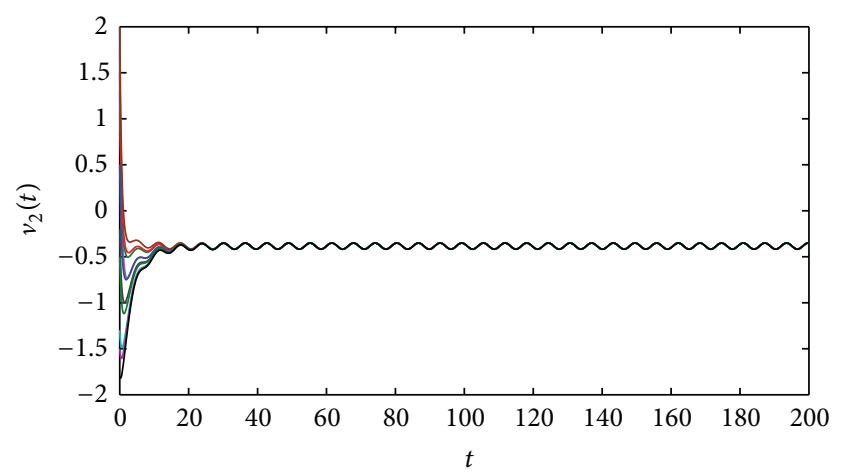

FIgURE 5: Transient response of state variables $v_{2}(t)$ of Example 1.

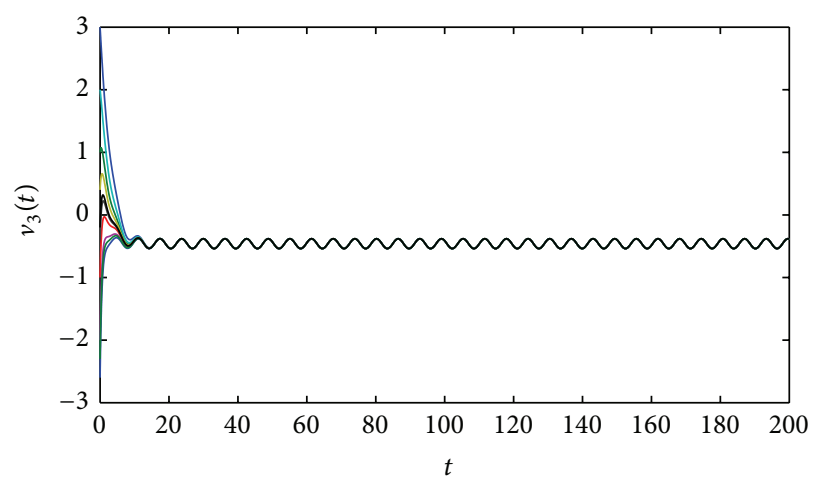

FIgURE 6: Transient response of state variables $v_{3}(t)$ of Example 1. 
On the other hand, we have the following results by simple calculation:

$$
\begin{aligned}
& \alpha_{1}-K_{1}=\frac{2}{5}>0, \quad \alpha_{2}-K_{2}=\frac{67}{80}>0 \\
& \alpha_{3}-K_{3}=\frac{14}{30}>0, \quad \beta_{1}-K_{1}^{*}=\frac{81}{70}>0, \\
& \beta_{2}-K_{2}^{*}=\frac{31}{24}>0, \quad \beta_{3}-K_{3}^{*}=\frac{7}{5}>0, \\
& \alpha_{1}-T_{1}-2+A_{1}\left(\sum_{j=1}^{3}\left|c_{1 j}\right| \bar{f}_{j}+\bar{I}_{1}\right)+\sum_{j=1}^{3} \bar{d}_{j} \frac{\left|h_{j 1}\right|}{1-\bar{\sigma}_{j 1}} k_{1} \\
& =-\frac{218.5}{240}<0 \\
& \alpha_{2}-T_{2}-2+A_{2}\left(\sum_{j=1}^{3}\left|c_{2 j}\right| \bar{f}_{j}+\bar{I}_{2}\right)+\sum_{j=1}^{3} \bar{d}_{j} \frac{\left|h_{j 2}\right|}{1-\bar{\sigma}_{j 2}} k_{2} \\
& =-\frac{237.1}{768}<0 \text {, } \\
& \alpha_{3}-T_{3}-2+A_{3}\left(\sum_{j=1}^{3}\left|c_{3 j}\right| \bar{f}_{j}+\bar{I}_{3}\right)+\sum_{j=1}^{3} \bar{d}_{j} \frac{\left|h_{j 3}\right|}{1-\bar{\sigma}_{j 3}} k_{3} \\
& =-\frac{7.19}{128}<0 \text {, } \\
& 2-\alpha_{1}-T_{1}+\bar{a}_{1} \sum_{j=1}^{3}\left|c_{1 j}\right| l_{j}+A_{1}\left(\sum_{j=1}^{3}\left|c_{1 j}\right| \bar{f}_{j}+\bar{I}_{1}\right) \\
& =-\frac{25.1}{48}<0 \text {, } \\
& 2-\alpha_{2}-T_{2}+\bar{a}_{2} \sum_{j=1}^{3}\left|c_{2 j}\right| l_{j}+A_{2}\left(\sum_{j=1}^{3}\left|c_{2 j}\right| \bar{f}_{j}+\bar{I}_{2}\right) \\
& =-\frac{23}{320}<0 \\
& 2-\alpha_{3}-T_{3}+\bar{a}_{3} \sum_{j=1}^{3}\left|c_{3 j}\right| l_{j}+A_{3}\left(\sum_{j=1}^{3}\left|c_{3 j}\right| \bar{f}_{j}+\bar{I}_{3}\right) \\
& =-\frac{1.85}{32}<0 \text {, } \\
& \beta_{1}-T_{1}^{*}-2+D_{1}\left(\sum_{i=1}^{3}\left|h_{1 i}\right| \bar{g}_{i}+\bar{J}_{1}\right)+\sum_{i=1}^{3} \bar{a}_{i} \frac{\left|c_{i 1}\right|}{1-\bar{\tau}_{i 1}} l_{1} \\
& =-\frac{2.3}{128}<0 \text {, } \\
& \beta_{2}-T_{2}^{*}-2+D_{2}\left(\sum_{i=1}^{3}\left|h_{2 i}\right| \bar{g}_{i}+\bar{J}_{2}\right)+\sum_{i=1}^{3} \bar{a}_{i} \frac{\left|c_{i 2}\right|}{1-\bar{\tau}_{i 2}} l_{2} \\
& =-\frac{85.1}{384}<0 \text {, }
\end{aligned}
$$

$$
\begin{aligned}
\beta_{3}-T_{3}^{*}-2+D_{3}\left(\sum_{i=1}^{3}\left|h_{3 i}\right| \bar{g}_{i}+\bar{J}_{3}\right)+\sum_{i=1}^{3} \bar{a}_{i} \frac{\left|c_{i 3}\right|}{1-\bar{\tau}_{i 3}} l_{3} \\
=-\frac{33.5}{128}<0, \\
2-\beta_{1}-T_{1}^{*}+\bar{d}_{j} \sum_{i=1}^{3}\left|h_{1 i}\right| k_{i}+D_{1}\left(\sum_{i=1}^{3}\left|h_{j i}\right| \bar{g}_{i}+\bar{J}_{1}\right) \\
=-\frac{87.9}{128}<0, \\
2-\beta_{2}-T_{2}^{*}+\bar{d}_{2} \sum_{i=1}^{3}\left|h_{2 i}\right| k_{i}+D_{2}\left(\sum_{i=1}^{3}\left|h_{2 i}\right| \bar{g}_{i}+\bar{J}_{2}\right) \\
=-\frac{83}{128}<0, \\
2-\beta_{3}-T_{3}^{*}+\bar{d}_{3} \sum_{i=1}^{3}\left|h_{3 i}\right| k_{i}+D_{3}\left(\sum_{i=1}^{3}\left|h_{3 i}\right| \bar{g}_{i}+\bar{J}_{3}\right) \\
=-\frac{41.55}{128}<0 .
\end{aligned}
$$

Then, the conditions of Theorem 3 hold. From Theorem 3 , system (86) has one $2 \pi$-periodic solution, and all other solutions of system (86) exponentially converge to it as $t \rightarrow$ $+\infty$.

Evidently, this consequence is coincident with the results of numerical simulation.

Example 2. For system (86), let $\alpha_{1}=2.12, \alpha_{2}=1.6, \alpha_{3}=1.7$, $\beta_{1}=2.2, \beta_{2}=5 / 3$, and $\beta_{3}=1.6$; the other parameters are the same as those in Example 1.

Through numerical simulation, Figures 7, 8, 9, 10, 11, and 12 depict the time responses of state variables of $u_{1}(t)$, $u_{2}(t)$, and $u_{3}(t)$ and $v_{1}(t), v_{2}(t)$, and $v_{3}(t)$, of the system in Example 2, respectively.

On the other hand, we have the following results by simple calculation:

$$
\begin{gathered}
\alpha_{1}-1-T_{1}=0.1>0, \quad \alpha_{2}-1-T_{2}=0.1>0, \\
\alpha_{3}-1-T_{3}=0.2>0, \quad \beta_{1}-1-T_{1}^{*}=0.2>0, \\
\beta_{2}-1-T_{2}^{*}=\frac{1}{3}>0, \quad \beta_{3}-1-T_{3}^{*}=0.1>0, \\
-T_{1}+A_{1} \sum_{j=1}^{3} \bar{f}_{j}\left|c_{1 j}\right|+A_{1} \bar{I}_{1}+\bar{a}_{1} \sum_{j=1}^{3} l_{j}\left|c_{1 j}\right|=-\frac{83}{96}<0, \\
-T_{2}+A_{2} \sum_{j=1}^{3} \bar{f}_{j}\left|c_{2 j}\right|+A_{2} \bar{I}_{2}+\bar{a}_{2} \sum_{j=1}^{3} l_{j}\left|c_{2 j}\right|=-\frac{77}{256}<0, \\
-T_{3}+A_{3} \sum_{j=1}^{3} \bar{f}_{j}\left|c_{3 j}\right|+A_{3} \bar{I}_{3}+\bar{a}_{3} \sum_{j=1}^{3} l_{j}\left|c_{3 j}\right|=-\frac{165}{640}<0, \\
-T_{1}^{*}+D_{1} \sum_{i=1}^{3} \bar{g}_{i}\left|h_{1 i}\right|+D_{1} \bar{J}_{1}+\bar{d}_{1} \sum_{i=1}^{3} k_{i}\left|h_{1 i}\right|=-\frac{115}{256}<0,
\end{gathered}
$$




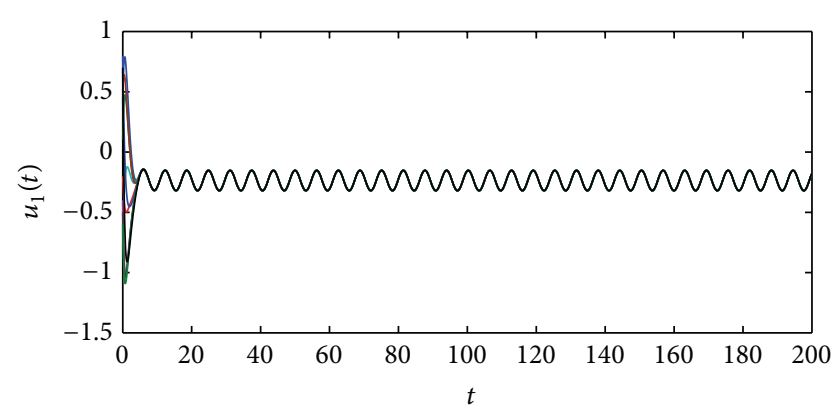

Figure 7: Transient response of state variables $u_{1}(t)$ of Example 2.

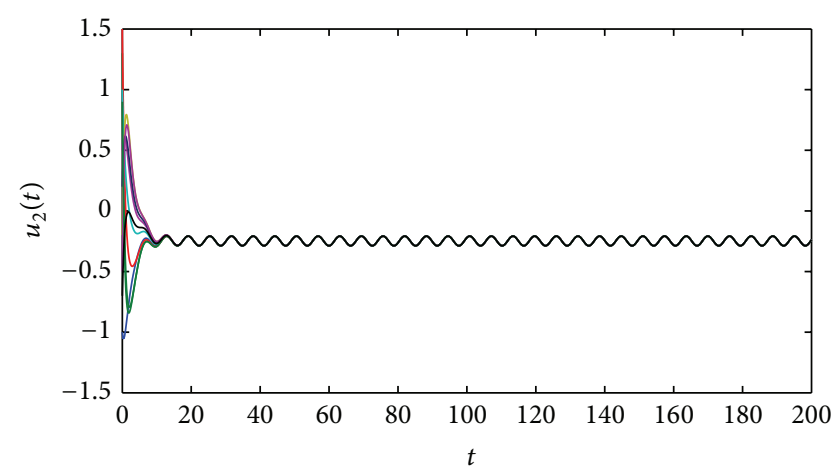

FIgURE 8: Transient response of state variables $u_{2}(t)$ of Example 2.

$$
\begin{aligned}
& -T_{2}^{*}+D_{2} \sum_{i=1}^{3} \bar{g}_{i}\left|h_{2 i}\right|+D_{2} \bar{J}_{2}+\bar{d}_{2} \sum_{i=1}^{3} k_{i}\left|h_{2 i}\right|=-\frac{83}{384}<0, \\
& -T_{3}^{*}+D_{3} \sum_{i=1}^{3} \bar{g}_{i}\left|h_{3 i}\right|+D_{3} \bar{J}_{3}+\bar{d}_{3} \sum_{i=1}^{n} k_{i}\left|h_{3 i}\right|=-\frac{99}{256}<0 .
\end{aligned}
$$

Then, the conditions of Theorem 4 hold. From Theorem 4 , system (86) has one $2 \pi$-periodic solution, and all other solutions of system (86) exponentially converge to it as $t \rightarrow+\infty$.

Evidently, this consequence is coincident with the results of numerical simulation.

Remark 5. Examples 1 and 2 showed that system (86) has one $2 \pi$-periodic solution, which is exponentially stable. In Example 1, there is

$$
\alpha_{1}-1-T_{1}=-0.3<0 .
$$

But this condition does not satisfy Theorem 4 . While in Example 2, there is

$$
\begin{gathered}
2-\beta_{3}-T_{3}^{*}+\bar{d}_{3} \sum_{i=1}^{3}\left|h_{3 i}\right| k_{i}+D_{3}\left(\sum_{i=1}^{3}\left|h_{3 i}\right| \bar{g}_{i}+\bar{J}_{3}\right) \\
=\frac{35.25}{128}>0 .
\end{gathered}
$$

This condition does not satisfy Theorem 3 . It showed that Theorems 3 and 4 have different applications.

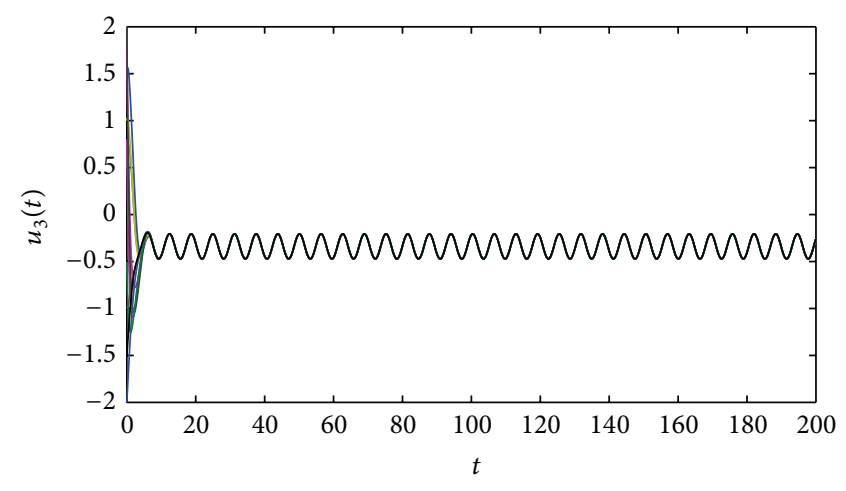

FIgURE 9: Transient response of state variables $u_{3}(t)$ of Example 2.

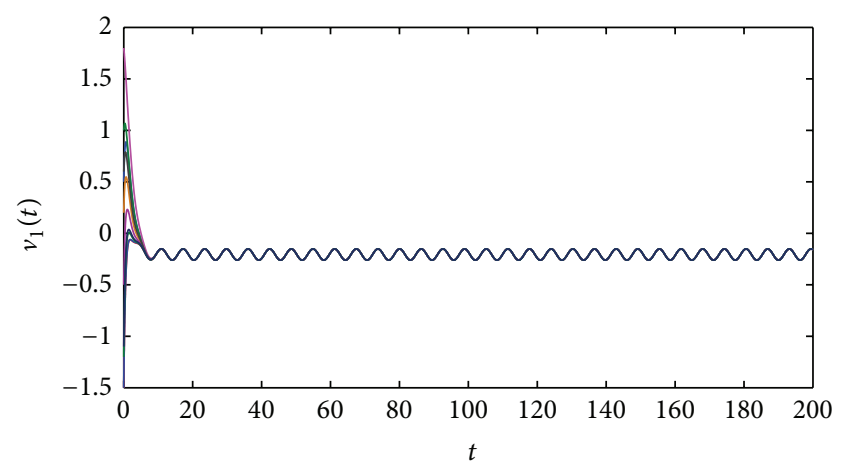

FIgUre 10: Transient response of state variables $v_{1}(t)$ of Example 2.

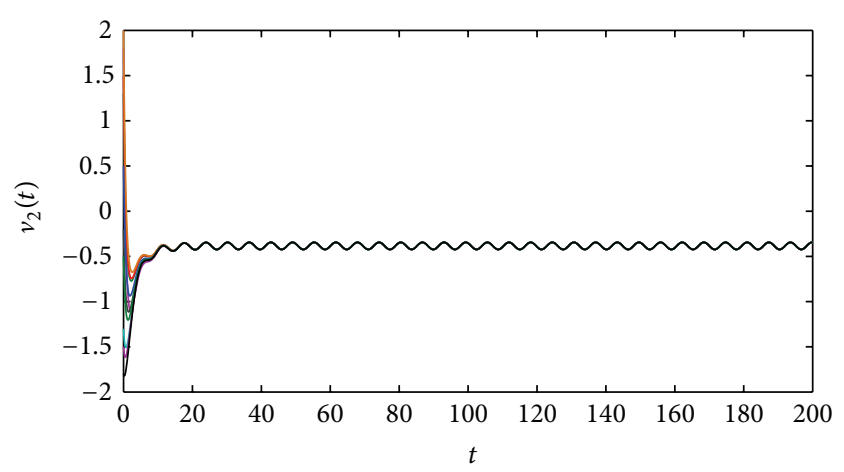

Figure 11: Transient response of state variables $v_{2}(t)$ of Example 2.

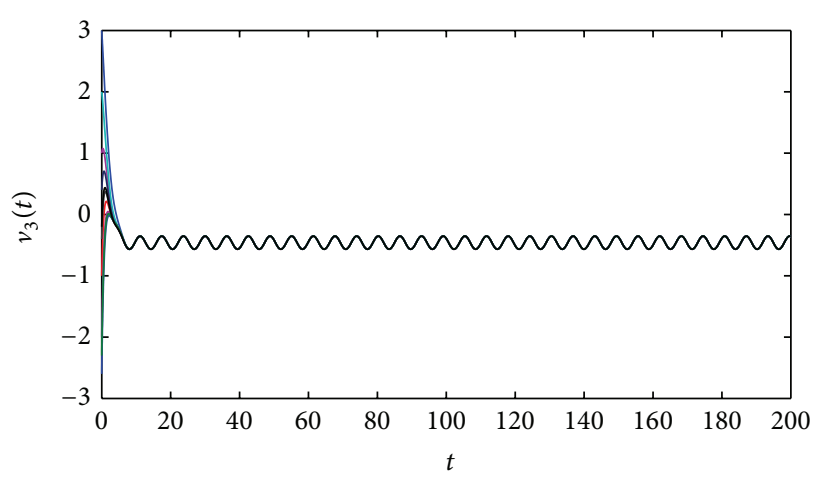

FIgURE 12: Transient response of state variables $v_{3}(t)$ of Example 2. 
In fact, the parameter $\alpha_{i}, \beta_{j}$ in Theorem 3 must be satisfied

$$
2-T_{i}<\alpha_{i}<2+T_{i}, \quad 2-T_{j}^{*}<\beta_{j}<2+T_{j}^{*} .
$$

For Theorem 4, it is only required to satisfy

$$
\alpha_{i}>1+T_{i}, \quad \beta_{j}>1+T_{j}^{*} .
$$

Therefore, Theorems 3 and 4 can solve different problems.

\section{Conclusion}

In this paper, we give three theorems to ensure the existence and the exponential stability of the periodic solution for inertial Cohen-Grossberg-type BAM neural networks. Novel existence and stability conditions are stated with simple algebraic forms and their verification and applications are straightforward and convenient. Especially, we give different conditions in Theorems 3 and 4 to ensure the exponential stability of the periodic solution, which have different advantages in different problems and applications. Finally two examples illustrate the effectiveness in different conditions. The method used in this paper can be employed to study general neural network with time-varying delays.

\section{Conflict of Interests}

The authors declare that there is no conflict of interests regarding the publication of this paper.

\section{Acknowledgment}

This work is supported by the Natural Science Foundation of Zhejiang Province (no. Y6100096).

\section{References}

[1] M. A. Cohen and S. Grossberg, "Absolute stability of global pattern formation and parallel memory storage by competitive neural networks," IEEE Transactions on Systems, Man and Cybernetics, vol. 13, no. 5, pp. 815-826, 1983.

[2] J. D. Cao and Q. Song, "Stability in Cohen-Grossberg-type bidirectional associative memory neural networks with timevarying delays," Nonlinearity, vol. 19, no. 7, pp. 1601-1617, 2006.

[3] F. Yang, C. Zhang, and D. Wu, "Global stability analysis of impulsive BAM type Cohen-Grossberg neural networks with delays," Applied Mathematics and Computation, vol. 186, no. 1, pp. 932-940, 2007.

[4] Q. Zhou and L. Wan, "Impulsive effects on stability of CohenGrossberg-type bidirectional associative memory neural networks with delays," Nonlinear Analysis. Real World Applications, vol. 10, no. 4, pp. 2531-2540, 2009.

[5] C. Bai, "Stability analysis of Cohen-Grossberg BAM neural networks with delays and impulses," Chaos, Solitons \& Fractals, vol. 35, no. 2, pp. 263-267, 2008.

[6] C. Feng and R. Plamondon, "Stability analysis of bidirectional associative memory networks with time delays," IEEE Transactions on Neural Networks, vol. 14, no. 6, pp. 1560-1565, 2003.
[7] H. Jiang and J. D. Cao, "BAM-type Cohen-Grossberg neural networks with time delays," Mathematical and Computer Modelling, vol. 47, no. 1-2, pp. 92-103, 2008.

[8] A. Chen and J. D. Cao, "Periodic bi-directional CohenGrossberg neural networks with distributed delays," Nonlinear Analysis. Theory, Methods \& Applications, vol. 66, no. 12, pp. 2947-2961, 2007.

[9] Y. Li and X. Fan, "Existence and globally exponential stability of almost periodic solution for Cohen-Grossberg BAM neural networks with variable coefficients," Applied Mathematical Modelling. Simulation and Computation for Engineering and Environmental Systems, vol. 33, no. 4, pp. 2114-2120, 2009.

[10] H. Xiang and J. D. Cao, "Exponential stability of periodic solution to Cohen-Grossberg-type BAM networks with timevarying delays," Neurocomputing, vol. 72, no. 7-9, pp. 1702-1711, 2009.

[11] C. G. Li, G. R. Chen, X. F. Liao, and J. B. Yu, "Hopf bifurcation and chaos in a single inertial neuron model with time delay," European Physical Journal B, vol. 41, no. 3, pp. 337-343, 2004.

[12] Q. Liu, X. F. Liao, G. Y. Wang, and Y. Wu, "Research for Hopf bifurcation of an inertial two-neuron system with time delay," in Proceedings of the IEEE International Conference on Granular Computing (GrC '06), pp. 420-423, May 2006.

[13] Q. Liu, X. F. Liao, D. G. Yang, and S. T. Guo, “The research for Hopf bifurcation in a single inertial neuron model with external forcing," in Proceedings of the IEEE International Conference on Granular Computing (GrC '07), pp. 528-533, November 2007.

[14] D. W. Wheeler and W. C. Schieve, "Stability and chaos in an inertial two-neuron system," Physica D, vol. 105, no. 4, pp. 267284, 1997.

[15] K. L. Babcock and R. M. Westervelt, "Stability and dynamics of simple electronic neural networks with added inertia," Physica D, vol. 23, no. 1-3, pp. 464-469, 1986.

[16] J. Ge and J. Xu, "Weak resonant double hopf bifurcations in an inertial four-neuron model with time delay," International Journal of Neural Systems, vol. 22, no. 1, pp. 63-75, 2012.

[17] Q. Liu, X. F. Liao, S. T. Guo, and Y. Wu, "Stability of bifurcating periodic solutions for a single delayed inertial neuron model under periodic excitation," Nonlinear Analysis. Real World Applications, vol. 10, no. 4, pp. 2384-2395, 2009.

[18] Q. Liu, X. F. Liao, Y. Liu, S. Zhou, and S. Guo, "Dynamics of an inertial two-neuron system with time delay," Nonlinear Dynamics, vol. 58, no. 3, pp. 573-609, 2009.

[19] H. Y. Zhao, L. Chen, and X.-H. Yu, "Bifurcation and control of a class of inertial neuron networks," Acta Physica Sinica, vol. 60, no. 7, Article ID 070202, 2011.

[20] Y. Q. Ke and C. F. Miao, "Stability analysis of BAM neural networks with inertial term and time delay," WSEAS Transactions on Systems, vol. 10, no. 12, pp. 425-438, 2011.

[21] Y. Q. Ke and C. F. Miao, "Stability and existence of periodic solutions in inertial BAM neural networks with time delay," Neural Computing and Applications, vol. 23, no. 3-4, pp. 10891099, 2012.

[22] Y. O. Horikawa and H. Kitajima, "Bifurcation and stabilization of oscillations in ring neural networks with inertia," Physica D, vol. 238, no. 23-24, pp. 2409-2418, 2009.

[23] Y. Q. Ke and C. F. Miao, "Stability analysis of inertial CohenGrossberg-type neural networks with time delays," Neurocomputing, vol. 117, no. 1, pp. 196-205, 2013. 


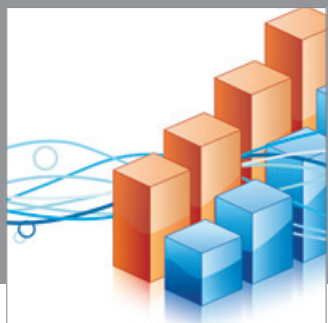

Advances in

Operations Research

mansans

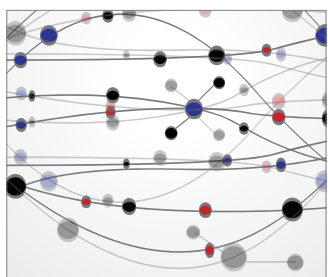

The Scientific World Journal
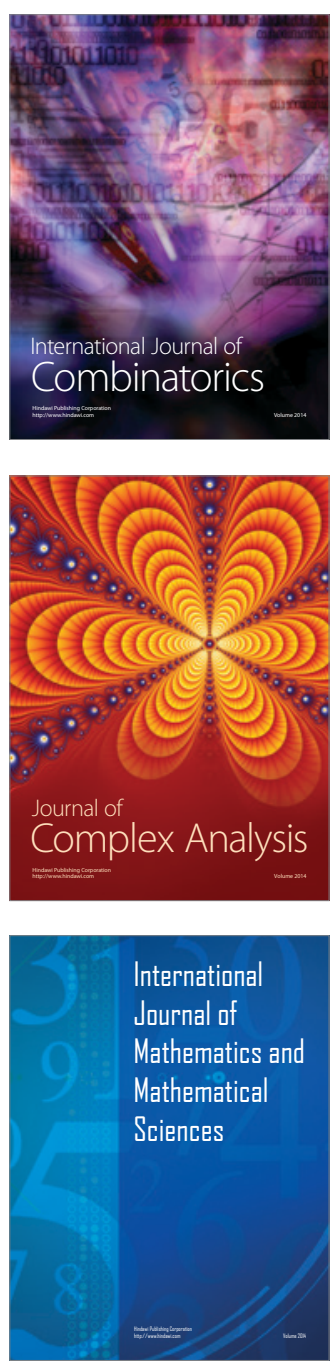
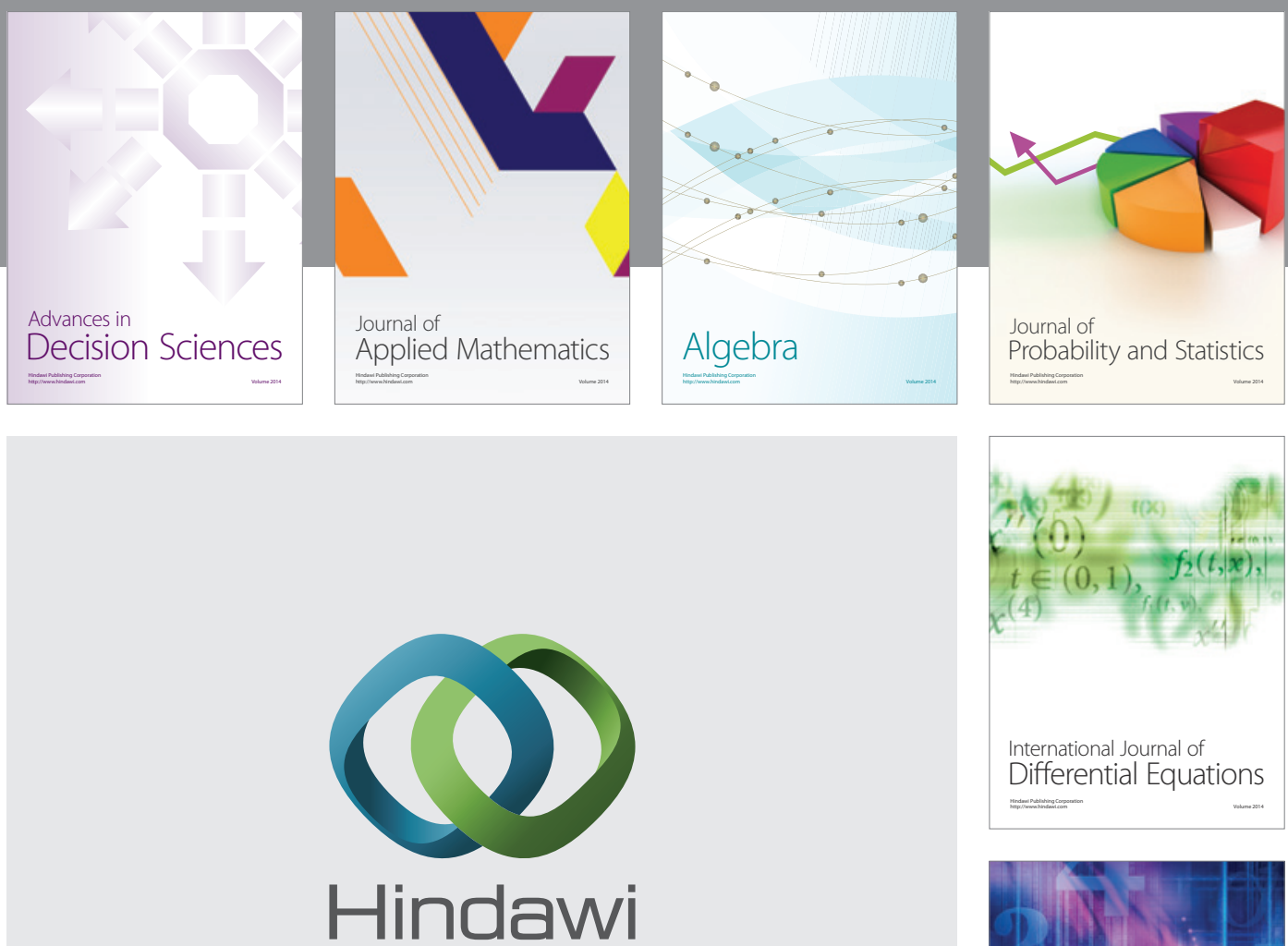

Submit your manuscripts at http://www.hindawi.com
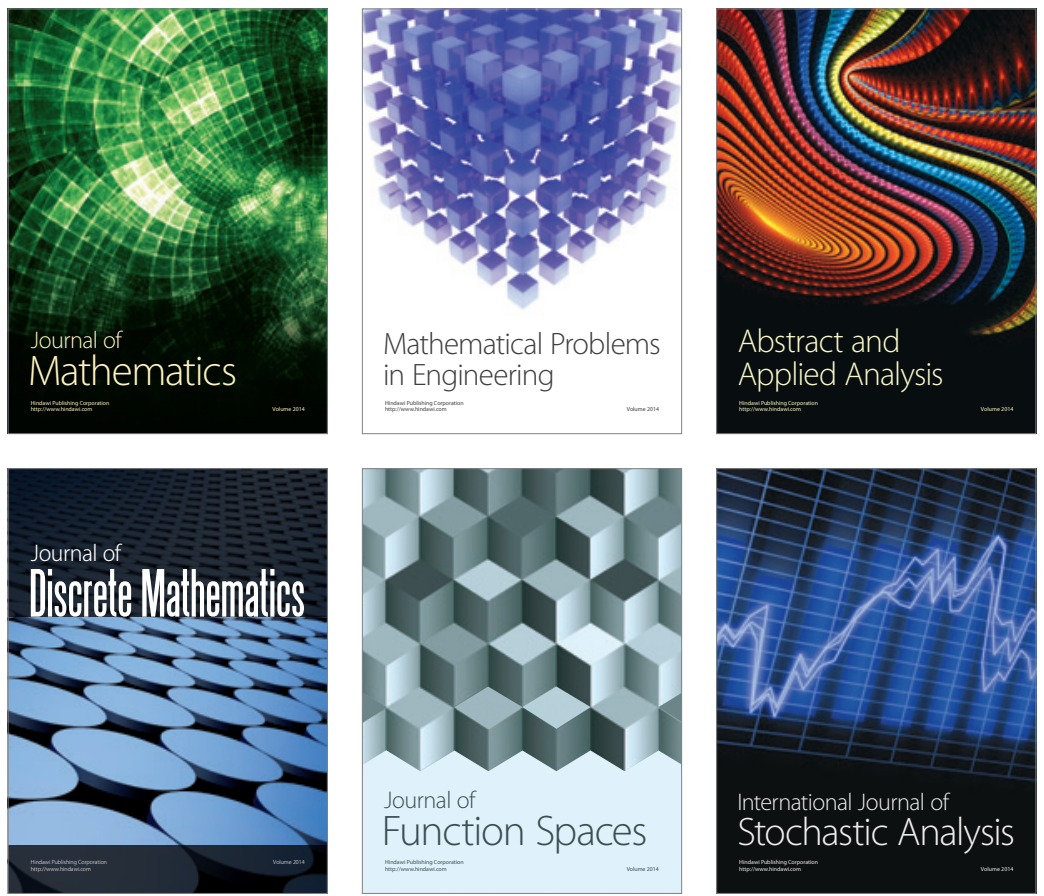

Journal of

Function Spaces

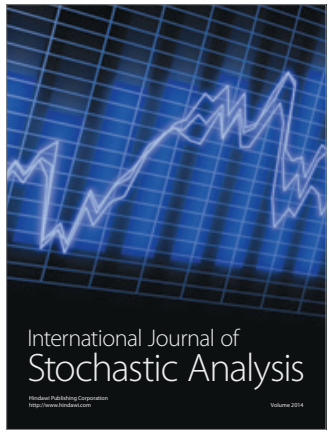

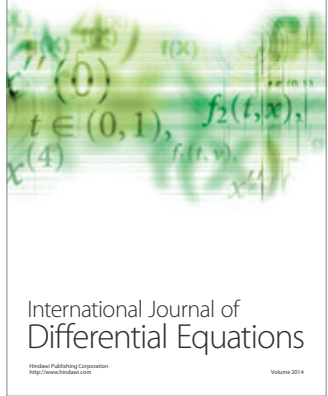
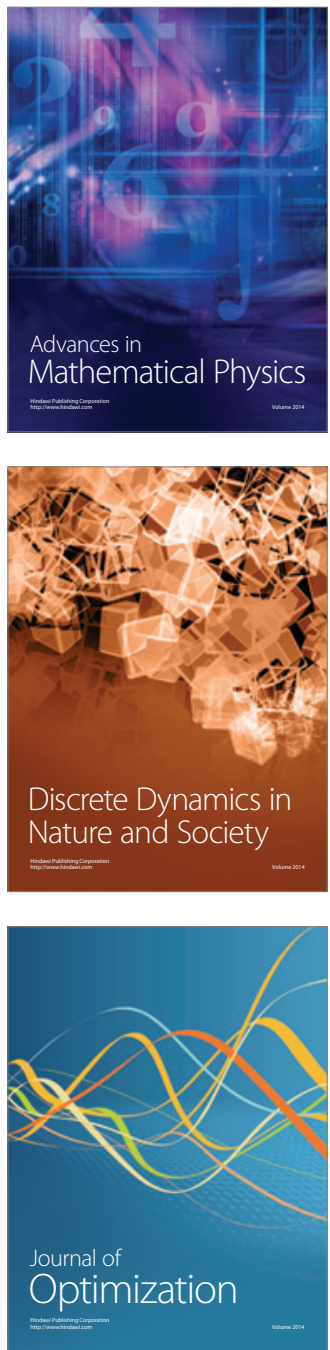